\begin{tabular}{|c|c|c|c|c|}
\hline \multicolumn{4}{|c|}{ НАУЧНО-ТЕХНИЧЕСКИЙ ВЕСТНИК ИНФОРМАЦИОННЫХ ТЕХНОЛОГИЙ, МЕХАНИКИ И ОПТИКИ } & \multirow{3}{*}{ 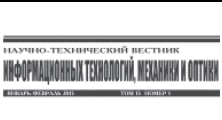 } \\
\hline 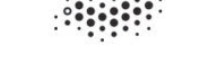 & $\begin{array}{cc}\text { март-апрель } 2016 & \text { Том } 16 \text { № } 2 \\
\text { SCIENTIFIC AND TECHNICAL JOURNAL O }\end{array}$ & $\begin{array}{l}\text { ISSN 2226-1494 } \\
\text { NFORMATION TEC }\end{array}$ & $\begin{array}{l}\text { http://ntv.ifmo.rul } \\
\text { IECHANICS AND OPTICS }\end{array}$ & \\
\hline УНИВЕРСИТЕТ ИТМО & March-April 2016 & ISSN 2226-1494 & http://ntv.ifmo.ru/en & \\
\hline
\end{tabular}

\author{
УДК 532.529 \\ ТЕНДЕНЦИИ РАЗРАБОТКИ ДЕТОНАЦИОННЫХ ДВИГАТЕЛЕЙ \\ ДЛЯ ВЫСОКОСКОРОСТНЫХ ВОЗДУШНО-КОСМИЧЕСКИХ ЛЕТАТЕЛЬНЫХ \\ АППАРАТОВ И ПРОБЛЕМА ТРОЙНЫХ КОНФИГУРАЦИЙ УДАРНЫХ ВОЛН \\ Часть II. Исследования встречных ударных волн и тройных \\ ударно-волновых конфигураций \\ П.В. Булат ${ }^{\text {, }}$ П.В. Денисенко \\ a Университет ИТМО, Санкт-Петербург, 197101, Российская Федерация \\ b Университет Уорвика, Ковентри, CV4 7AL, Великобритания \\ Адрес для переписки: pavelbulat@mail.ru \\ Информация о статье \\ Поступила в редакцию 29.12.15, принята к печати 24.02 .16 \\ doi: 10.17586/2226-1494-2016-16-2-199-223 \\ Язык статьи - русский
}

Ссылка для цитирования: Булат П.В., Денисенко П.В. Тенденции разработки детонационных двигателей для высокоскоростных воздушно-космических летательных аппаратов и проблема тройных конфигураций ударных волн. Часть II. Исследования встречных ударных волн и тройных ударно-волновых конфигураций // Научно-технический вестник информационных технологий, механики и оптики. 2016. Т. 16. № 2. С. 199-223. doi: 10.17586/2226-1494-2016-16-2-199-223

\title{
Аннотация
}

Рассматриваются актуальные задачи развития теории интерференции газодинамических разрывов в приложении к задаче совершенствования силовых установок воздушно-космических летательных аппаратов, рассчитанных на большие сверхзвуковые скорости полета. В первой части обзора была изложена история изучения детонации и различные концепции детонационных двигателей, а также воздухозаборников, рассчитанных на гиперзвуковые скорости полета. Во второй части приведен обзор работ по развитию теории интерференции газодинамических разрывов. Приведена классификация газодинамических разрывов, ударно-волновых процессов, ударно-волновых структур, тройных конфигураций ударных волн. Показано, что многие из этих процессов сопровождаются явлением гистерезиса, имеются области неоднозначности, следовательно, при проектировании двигателей и воздухозаборников необходимо уметь создавать оптимальные ударно-волновые структуры и обеспечивать их устойчивость. Большое внимание в последнее время уделяется использованию в воздухозаборниках ударно-волновых структур, с переотражением скачков уплотнения и интерференцией скачков уплотнения противоположных направлений. В настоящем обзоре им уделено основное внимание, приведены ссылки на этапные работы, последние расчетные и экспериментальные результаты. К сожалению, в зарубежных обзорах пропущены многие этапные работы советских и российских исследователей, так как они не были опубликованы на английском языке. В то же время, именно советская школа газовой динамики сформулировала теорию интерференции газодинамических разрывов в современном виде. Одной из целей настоящего обзора является восполнение этого пробела. Обзор может быть рекомендован специалистам, инженерам и научным сотрудникам, работающим в области аэрокосмической техники.

Ключевые слова

ударная волна, ударно-волновые структуры, тройные конфигурации ударных волн, встречные ударные волны

\section{TRENDS IN THE DEVELOPMENT OF DETONATION ENGINES FOR HIGH-SPEED AEROSPACE AIRCRAFTS AND THE PROBLEM OF TRIPLE CONFIGURATIONS OF SHOCK WAVES. \\ Part II - Research of Counterpropagating Shock Waves and Triple Shock Wave Configurations}

P.V. Bulat ${ }^{a}$, P.V. Denissenko

a ITMO University, Saint Petersburg, 197101, Russian Federation

${ }^{\mathbf{b}}$ Warwick University, Coventry, CV4 7AL, United Kingdom

Corresponding author: pavelbulat@mail.ru

Article info

Received 29.12.15, accepted 24.02.16

doi: 10.17586/2226-1494-2016-16-2-199-223

Article in Russian

For citation: Bulat P.V., Denissenko P.V. Trends in the development of detonation engines for high-speed aerospace aircrafts and the problem of triple configurations of shock waves. Part II - Research of counterpropagating shock waves and triple shock wave configurations. Scientific and Technical Journal of Information Technologies, Mechanics and Optics, 2016, vol. 16, no. 2, pp. 199-223. doi: 10.17586/2226-1494-2016-16-2-199-223 


\begin{abstract}
The paper deals with current issues of the interference theory development of gas-dynamic discontinuities as applied to a problem of propulsion refinement for the air-spacecrafts, designed for hypersonic flight speeds. In the first part of the review we have presented the history of detonation study and different concepts of detonation engines, as well as air intakes designed for hypersonic flight speeds. The second part provides an overview of works on the interference theory development for gasdynamic discontinuities. We report about classification of the gas-dynamic discontinuities, shock wave propagation, shock-wave structures and triple configurations of shock waves. We have shown that many of these processes are accompanied by a hysteresis phenomenon, there are areas of ambiguity; therefore, in the design of engines and air intakes optimal shock-wave structures should be provided and their sustainability should be ensured. Much attention has recently been given to the use of the air intakes in the shock-wave structures with the rereflection of shock waves and the interference of shock waves in the opposite directions. This review provides increased focus on it, contains references to landmark works, the last calculated and experimental results. Unfortunately, foreign surveys missed many landmark works of the Soviet and Russian researchers, as they were not published in English. At the same time, it was the Soviet school of gas dynamics that has formulated the interference theory of gas-dynamic discontinuities in its present form. To fill this gap is one of this review scopes. The review may be recommended for professionals, engineers and scientists working in the field of aerospace engineering.
\end{abstract}

Keywords

shock-wave, shock-wave structures, triple shock-wave configurations, counterpropagating shock waves

\title{
Введение
}

Большинство задач сверхзвуковой аэрогазодинамики связано с ударно-волновыми процессами (УВП), которые представляют собой объект исследований. Создание физико-математических моделей УВП - предмет исследований. Представления о волнах как средстве распространения звука были известны еще в античной Греции. Построение математических моделей этих волн началось с работ Пуассона, было продолжено Риманом и привело в середине XIX века, благодаря стараниям Стокса, Рэнкина, Гюгонио и других ученых этого и следующих столетий, к моделям ударных волн.

В.Н. Усков в ряде обзоров - историческом очерке «Наука о стихиях» [1], обзоре истории развития аэрогазодинамики [2], истории исследования ударных волн [3] и обзорах работ отечественных аэрогазодинамиков $[4,5]$ - привел достаточно подробные сведения об основных этапах прогресса в области исследования УВП. В различных (в том числе неопубликованных) работах В.Н. Ускова рассмотрены вопросы классификации газодинамических разрывов (ГДР), ударно-волновых процессов, задачи управления струйными и ударно-волновыми процессами.

В 2001 г. вышел трехтомник, посвященный ударным волнам, под редакцией Бен-Дора [6]. В первом томе этого издания, претендующего на всеобъемлющее описание предметной области, связанной с ГДР, приведен подробный перечень работ, в которых, начиная с XVII века, формировались представления о волновых процессах в природе. Особенно подробно рассмотрен ранний период - до 30-х годов $\mathrm{XX}$ века. Однако, несмотря на большой объем представленного материала, многие практически важные задачи не освещены. Отсутствуют полнота и единство классификации волн и разрывов. Необходимо также восполнить пробел, связанный с вкладом советских и российских математиков в развитие теории интерференции ударных волн и волновых фронтов, так как зарубежные обзоры традиционно невнимательно относятся к работам советской и российской школы, отмечая только публикации, переведенные на английский язык. Наибольший вклад в развитие теории интерференции ГДР внесла ленинградская научная школа газовой динамики под руководством сначала И.П. Гинзбурга, а затем - В.Н. Ускова. Работы велись в Санкт-Петербургском государственном университете (ранее - Ленинградский государственный университет) и Балтийском государственном техническом университете «Военмех» (ранее - Ленинградский механический институт). Другим важнейшим центром изучения интерференции ударных волн стал Новосибирск. Работы в этом направлении велись в Институте теоретической и прикладной механики им. С.А. Христиановича (ИТПМ) и в Институте гидродинамики им. М.А. Лаврентьева.

\section{Газодинамические разрывы и процессы}

\section{Понятие о волнах, газодинамических разрывах и ударно-волновых процессах}

Ударно-волновыми процессами называются процессы преобразования газодинамической системы, имеющей параметры $f$, в систему с параметрами $\hat{f}$.

$$
f \rightarrow \hat{f} .
$$

Здесь $f$ и $\hat{f}$ - множества газодинамических переменных до и после УВП. Эти множества включают кинематические, термодинамические и теплофизические переменные, характеризующие параметры потока газа: кинематические ( $u$ - скорость, $w$ - ускорение); термодинамические ( $p$ - давление, $\rho$ - плотность, $T$ - температура); $f_{0}$ - соответствующие параметры торможения; изменение энтропии $\Delta S=C_{V} \ln \vartheta / \vartheta$, где $\vartheta=p / \rho^{\gamma}-$ инвариант Лапласа-Пуассона; изменение $h$ и $h_{0}$ - энтальпии; а также теплофизических параметров (теплоемкости $c_{p}$ и $c_{V}$, показатель адиабаты $\gamma$, вязкости $v$ и пр.), которые могут изменяться в ходе УВП. 
Интенсивность УВП, как правило, характеризуется отношением статических давлений после и до УВП. Часто также используется обозначение $J=p_{1} / p$, где $p_{1}-$ давление после УВП. Значения $J>1$ характеризуют уплотнение потока, а величины $J<1$ - его расширение (разряжение).

Газодинамические разрывы представляют собой некоторую идеализацию области с резким изменением параметров $f$, заменяя ее поверхностями, на которых газодинамические переменные изменяются скачком.

Порядок газодинамического разрыва. ГДР в сверхзвуковых течениях бывают нулевого порядка $\Phi_{0}$ - центр волны разрежения/сжатия, скачок уплотнения и поверхность скольжения, на которых терпят разрыв газодинамические параметры течения (давление $p$, полное давление $p_{0}$, скорость $u$, угол наклона вектора скорости $\vartheta$ ); и первого порядка $\Phi_{1}$, называемые также слабыми разрывами (разрывные характеристики, слабые тангенциальные разрывы), на которых терпят разрыв первые производные газодинамических переменных. Можно определить особенности (разрывы) $\Phi_{i}$ пространства газодинамических переменных любого порядка.

Отличие волн от разрывов заключается в том, что волны имеют конечную ширину и занимают область изменения газодинамических переменных от значений $f$ до $\hat{f}$, заключенную между передним и задним фронтами волны.

Волны и разрывы некоторых типов (но не всех) также являются УВП.

Ударные волны $\mathbf{D}_{0}$ относятся к газодинамическим разрывам, они могут зарождаться внутри исходного потока с известными параметрами $f$ либо вноситься в поток извне. Моделью ударных волн является поверхность математического разрыва первого рода, при переходе через которую газодинамические переменные терпят разрыв $[f]=\hat{f}-f \neq 0$. Частным случаем D-волн (ударных волн) являются стоячие $\mathbf{D}_{0}$ в сверхзвуковом исходном потоке (число Маха $M=u / a>1, a-$ скорость звука) ударные волны, когда скорость волны $\mathbf{D}_{0}=0$. Такие стоячие волны часто называют скачками уплотнения $\boldsymbol{\sigma}$. Скачков и ударных волн разрежения не бывает.

Реальная ширина ударной волны, как показал Прандтль [7], соизмерима с длиной $\lambda$ свободного пробега молекул и может быть довольно большой в разреженных газах. В плотных средах она незначительна и модель математического разрыва нулевой протяженности вполне адекватна. Первым сумел получить фотографию ударной волны в 1889 г. Эрнст Мах [8].

Изоэнтропические волны представляют собой звуковые (акустические) поля, а также простые волны Римана (R) в нестационарных движениях газа и волны Прандтля-Майера (罗) в сверхзвуковых стационарных плоских потоках. Принято разделять волны разряжения, в которых отношение статических давлений $J_{r} \equiv \hat{p} / p<1$, и волны уплотнения (сжатия), в которых $J_{c}>1$.

Тангенциальные $\tau$ и контактные К (энтропийные) разрывы не пересекаются линиями тока. В тангенциальном разрыве вектор скорости коллинеарен плоскости разрыва, а в контактном нет. Через тангенциальные и контактные разрывы газ не перетекает, эти разрывы разделяют движущиеся потоки газов с различными термодинамическими переменными, за исключением статических давлений. Таким образом, тангенциальные и контактные разрывы не являются УВП.

\section{Развитие представлений о природе газодинамических разрывов}

Дифференциальные уравнения относительно плотности $\rho$ и потенциала скорости $\varphi$, описывающие одномерные нестационарные движения невязкого совершенного изотермического газа, впервые представлены в 1788 г. в книге Лагранжа [9], обозначения - как в оригинале:

$$
\begin{aligned}
& a^{2} \ln \frac{\rho}{D}+\frac{\partial \varphi}{\partial t}+\frac{1}{2}\left(\frac{\partial \varphi}{\partial x}\right)^{2}=0 ; \\
& \frac{\partial \rho}{\partial t}+\rho \frac{\partial^{2} \varphi}{\partial x^{2}}+\frac{\partial \rho}{\partial x} \frac{\partial \varphi}{\partial x}=0 .
\end{aligned}
$$

Здесь $a$ - скорость звука, $D$ - плотность первоначально невозмущенной среды, $x$ - координата, $t-$ время. Для связи между давлением, плотностью и скоростью звука Лагранж использовал соотношение, ранее предложенное Ньютоном: $p=a^{2} \rho$.

В 1808 году Пуассон [10] получил выражение в виде плоской волны:

$$
\frac{\partial \varphi}{\partial x}=F\left[x+t\left(a-\frac{\partial \varphi}{\partial x}\right)\right],
$$

где $F$ - некоторая произвольная функция, определяемая начальными и (или) граничными условиями. В 1848 г. Стокс [11] обратил внимание на то, что решения этих уравнений остаются непрерывными только в течение ограниченного промежутка времени. Интересно, что именно в такой постановке задача изуче- 
ния движения идеального газа, содержащего разрывы, рассматривалась в конце XX века научной школой советского математика В.И. Арнольда [12].

Уравнение Пуассона описывает поле скоростей свободно движущихся по прямой частиц. Закон свободного движения частицы имеет вид $x=\varphi(t)=x_{0}+u t$, где $u-$ скорость частицы. Функция $\varphi$ удовлетворяет уравнению Ньютона. По определению $d \varphi / d t=u(t, \varphi)$. Продифференцировав последнее соотношение по $t$, приходим к уравнению, получившему название уравнения Эйлера:

$$
u \frac{\partial u}{\partial x}+\frac{\partial u}{\partial t}=0
$$

Таким образом, описания движения при помощи уравнения Эйлера для поля газодинамических переменных и при помощи уравнения Ньютона для частиц эквивалентны. Известно, что квазилинейные дифференциальные уравнения в частных производных решаются с помощью построения характеристик. Характеристики уравнения Эйлера эквивалентны закону Ньютона для движущейся частицы [13], и задачу о распространении волны можно решить путем построения характеристик, вдоль которых движутся материальные частицы. На рис. 1 показано, как решается уравнение Эйлера с помощью характеристик.

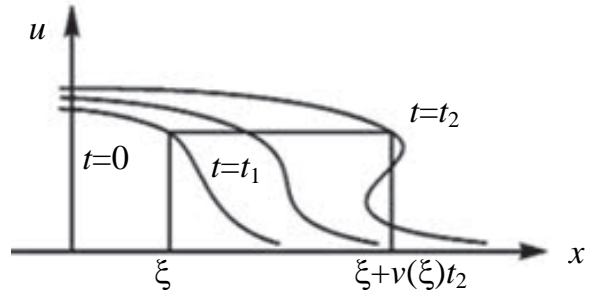

a

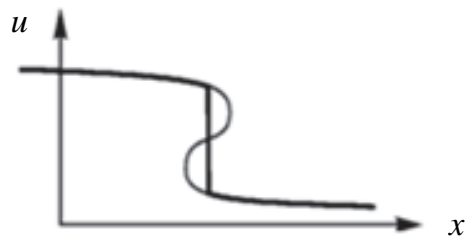

6

Рис. 1. Решение уравнения Эйлера с помощью характеристик (а) и появление разрыва в решении (б): $\xi$ - координата выбранной материальной точки с заданной скоростью $u$

На плоскости $y-x$ задана начальная функция $y=u_{0}(x)_{t=0}$. Уравнения характеристик $t^{\prime}=1, y^{\prime}=0, x^{\prime}=y$. Если отложить от этой кривой горизонтальные линии, то вдоль каждой из них частицы будут двигаться со своей постоянной скоростью. Тогда в некоторые моменты времени $t=t_{1}, t_{2} \ldots t_{n}$ форма распределения скорости $u(x)$ будет меняться.

В некоторый момент времени $\left(t_{2}\right)$ отображение $u(x)$ перестает быть графиком функции, т.е. имеются значения $x$, которым соответствует несколько значений $и$. В этой области физическое условие отсутствия взаимодействия частиц означает их прохождение сквозь друг друга, что нефизично. Нужно вводить некоторую модель их взаимодействия. Например, в модели образования Вселенной, предложенной Я.Б. Зельдовичем [14], учитывается расширение Вселенной и гравитационное взаимодействие. Добавление таких условий приводит к появлению особенностей в решении, т.е. областей, где концентрация частиц (у Я.Б. Зельдовича - галактик) максимальна. Такие области (множество критических значений) называются каустиками.

В рассматриваемом нами примере сверхзвукового течения идеального газа необходимо ввести модель неупругого столкновения частиц. Тогда в месте этого столкновения будет образовываться ударная волна - разрыв параметров движения частиц (рис. 1, б). В решении появляются разрывы, так как уравнение поля Эйлера перестает однозначно описывать распределение газодинамических переменных. Стокс в упомянутом выше труде первым ввел понятие разрыва (discontinuity) и получил два условия для плотности $\rho$ и скорости газа и по сторонам разрыва, следующие из законов сохранения массы и количества движения:

$$
\begin{aligned}
& \rho_{1} u_{1}-\rho_{2} u_{2}=\left(\rho_{1}-\rho_{2}\right) \vec{V} ; \\
& \left(\rho_{1} u_{1}-\rho_{2} u_{2}\right) \vec{V}-\left(\rho_{1} u_{1}^{2}-\rho_{2} u_{2}{ }^{2}\right)=a^{2}\left(\rho_{1}-\rho_{2}\right) .
\end{aligned}
$$

Здесь $\vec{V}$ - скорость распространения разрыва, индексом «2» обозначены параметры за разрывом, индексом «1»- параметры до разрыва.

Такие разрывы, на которых газодинамические параметры меняются скачком, впоследствии назвали сильными. Стокс отметил, что скачкообразное изменение параметров на разрывах является результатом пренебрежения вязкостью и теплопроводностью среды.

Простейшей моделью неупругого столкновения частиц является уравнение Бюргерса [15], которое описывает газодинамическое поле в гладких областях пространства и взаимодействие частиц газа внутри ударной волны:

$$
\frac{\partial u}{\partial t}+u \frac{\partial u}{\partial x}=\varepsilon \frac{\partial^{2} u}{\partial x^{2}} .
$$


При малой вязкости $\varepsilon$ оно приближает уравнение Эйлера в областях плавного изменения параметров. Ирншоу $[16,17]$ рассмотрел одномерные нестационарные течения газа - как изотермические, так и адиабатические. Им были получены решения в виде плоской волны, в которой с течением времени образуются области резкого изменения параметров, которые он, как и Стокс, назвал разрывами. Важную роль в анализе ГДР, сверхзвуковых движений газа играет скорость распространения возмущений - скорость звука. Ирншоу ввел следующую связь между давлением, плотностью и скоростью звука:

$$
a=\sqrt{k \mu}, \mu=p / \rho,
$$

где $k$ - отношение удельной теплоемкости газа при постоянном давлении к удельной теплоемкости при постоянном объеме. Сейчас стандартным считается обозначение $\gamma$.

\section{Условия динамической совместности на разрывах и волнах}

Соотношения переменных $\hat{f}$ и $f$ по разные стороны ГДР получили название условий динамической совместимости (УДС) [18]. УДС на стационарных разрывах представляют собой балансы удельных потоков

- вещества

$$
\left[\rho u_{n}\right]=\hat{\rho} \hat{u}_{n}-\rho u_{n}=0,
$$

- нормальной компоненты импульса

$$
\left[p+\rho u_{n}^{2}\right]=0,
$$

- тангенциальной компоненты импульса

$$
\left[\rho u_{n} u_{\tau}\right]=0,
$$

- энергии

$$
\left[\rho u_{n} h_{0}\right]=0,
$$

где $u_{n}$ и $u_{\tau}$ - проекции вектора скорости на направления, нормальное к плоскости разрыва и параллельное ей. Из приведенной системы несложно получить адиабату Лапласса-Пуассона (изоэнтропу) [19],

$$
J E^{\gamma}=1
$$

и ударную адиабату Рэнкина-Гюгонио,

$$
E=\frac{1+\varepsilon J}{J+\varepsilon},
$$

где $\varepsilon=(\gamma-1) /(\gamma+1), E=\rho / \hat{\rho}, J=\hat{p} / p-$ интенсивность ударно-волнового процесса уплотнения $(J>1)$ или разрежения $(J<1)$. Числа Маха по разные стороны волны или разрыва связаны формулой

$$
\frac{\mu}{\hat{\mu}}=E J \text {, }
$$

где $\mu=\left(1+\varepsilon\left(M^{2}-1\right)\right.$ и $\hat{\mu}=1+\varepsilon\left(\hat{M}^{2}-1\right)$. В зависимости от того, какую формулу для адиабаты использовать - (3) или (2), формула (4) дает возможность определить числа Маха за волнами разрежения/сжатия и скачками уплотнения. Дополняя приведенные соотношения уравнением состояния газа, можно получить в удобном виде отношения основных газодинамических переменных до и после волны или разрыва,

$$
M_{2}^{2}=\frac{M^{2}-(1-E)(J+1)}{E J},
$$

отношение температур,

$$
\frac{T_{2}}{T}=E J,
$$

отношение скоростей звука,

$$
\frac{a_{2}}{a}=\sqrt{E J},
$$

коэффициент восстановления полного давления,

$$
I_{0}=\frac{p_{02}}{p_{01}}=\left(E^{\gamma} J\right)^{\frac{-1}{\gamma-1}},
$$

отношение плотностей,

$$
\frac{\rho_{2}}{\rho_{1}}=\frac{1}{E} \text {. }
$$

Индекс «2» соответствует параметрам за волной или разрывом, индекс «1»- до волны или разрыва. Записанные в таком виде соотношения справедливы для любых типов волн - простых, ударных и де- 
тонационных. Если вместо E в соотношения (5)-(9) подставить уравнения адиабаты Лапласа-Пуассона (2), то получим соотношения для простых и центрированных изоэнтропических волн, если подставить адиабату Рэнкина-Гюгонио (3), то - уравнения для ударных волн. Все переменные за скачком в уравнениях (5)-(9) монотонно изменяются в зависимости от интенсивности скачка $J$. Соотношения (5)-(9) называются условиями динамической совместности в форме обобщенной адиабаты [20]. В столь удобном для построения методик расчета универсальном виде их, видимо, первым получил В.Н. Усков [21].

В законченном виде УДС появились далеко не сразу. Сформулированных Стоксом условий (1) недостаточно, чтобы определить два неизвестных параметра потока за разрывом и скорость распространения самого разрыва. Первой попыткой замкнуть написанную Стоксом систему уравнений была опубликованная в 1860 г. работа Римана [22]. В этой работе автор предположил, что при переходе через нормальный разрыв энтропия постоянна, и дополнил систему Стокса (1) третьим уравнением. Объяснить возникающее при этом предположении изменение энергии при переходе через разрыв Риман не смог. Независимо от Римана, Рэнкин в 1869-1870 г.г. [23-25] получил третье уравнение, дополняющее систему (1), в другом виде. Он установил связь между параметрами по сторонам ударной волны, рассмотрев непрерывно меняющиеся внутри нее состояния среды, в которой происходит равновесный теплообмен. Суммарное количество теплоты, полученное средой, должно быть равно нулю. Используя соотношения равновесной термодинамики и формулы Стокса, Рэнкин получил выражения для скорости распространения нормального разрыва по неподвижной среде,

$$
a^{2}=S\left\{(\gamma+1) \frac{p}{2}+(\gamma-1) \frac{P}{2}\right\},
$$

(не путать со скоростью звука «а») и скорости потока за ним,

$$
u=(p-P) \frac{S}{a},
$$

через известные давления $P$ до разрыва и за ним $p$, а также известный удельный объем до разрыва $S$ для совершенного газа (обозначения даны как в оригинале, сейчас более распространены другие обозначения).

Наиболее важным результатом, следующим из соотношений (10)-(11) Рэнкина, является утверждение, что нормальные разрывы всегда распространяются относительно неподвижной среды со сверхзвуковой скоростью, в то время как относительно среды за разрывом их скорость распространения всегда дозвуковая. Способ получения УДС на ударной волне, примененный Рэнкиным, приводит к выполнению всех законов сохранения, но он учитывает теплопроводность газа, а его вязкостью пренебрегает, что не слишком обосновано, так как вязкость и теплопроводность взаимосвязаны. Гюгонио получил условие на нормальном разрыве более строго, чем Рэнкин, как следствие закона сохранения энергии, минуя рассмотрение состояния газа «внутри» ударной волны [26]. Это условие совпадает с полученным ранее условием Рэнкина, но для его вывода Гюгонио не потребовалось дополнительных предположений. В результате УДС на ударной волне получили названия условия Рэнкина-Гюгонио.

\section{Косой скачок уплотнения и ударные поляры}

Подробный анализ газодинамических волн (изоэнтропических волн разрежения и сжатия) и косых скачков уплотнения, возникающих в плоских стационарных течениях невязкого нетеплопроводного совершенного газа, был опубликован в 1908 г. Майером [27]. В этой же работе определены параметры косого скачка уплотнения, образующегося при обтекании плоского острого угла. Это задача является важной для практики, так как обтекание наклонной преграды - одна из часто встречающихся причин появления скачка уплотнения в потоке газа. Начиная с этой работы Майера, в качестве основного параметра, характеризующего скачок уплотнения, рассматривают его интенсивность - отношение статических давлений по его сторонам. В современном виде УДС на скачках уплотнения были сформулированы В.Н. Усковым в 1980 г. В дальнейшем они были развиты на случай одномерных бегущих волн [28], а также косых бегущих ударных волн. Угол наклона скачка $\sigma$, его интенсивность $J$ и угол отклонения потока на скачке $\beta$ при заданных параметрах течения перед скачком $\left(M_{1}, p_{1}, p_{01}, \rho\right)$ взаимно однозначно связаны между собой. Задание любого из этих трех параметров позволяет вычислить два других,

$$
J_{\sigma}=(1+\varepsilon) M^{2} \sin ^{2} \sigma-\varepsilon,
$$

а также связь между углами поворота потока $\beta$ и наклона скачка $\sigma$,

$$
\operatorname{tg} \beta=\frac{M^{2} \sin ^{2} \sigma-1}{\frac{1}{1-\varepsilon} M^{2}-\left(M^{2} \sin ^{2} \sigma-1\right)} \operatorname{ctg} \sigma .
$$

Уравнения (12)-(13) определяют при заданном $M$ ударную поляру $\ln J-\beta$, (рис. 2) в параметрической форме с параметром $\sigma$, который может меняться в пределах от угла Маха $\alpha=\arcsin (1 / M)$ до $90^{\circ}$. За характерную форму ударные поляры называют также сердцевидными кривыми. 


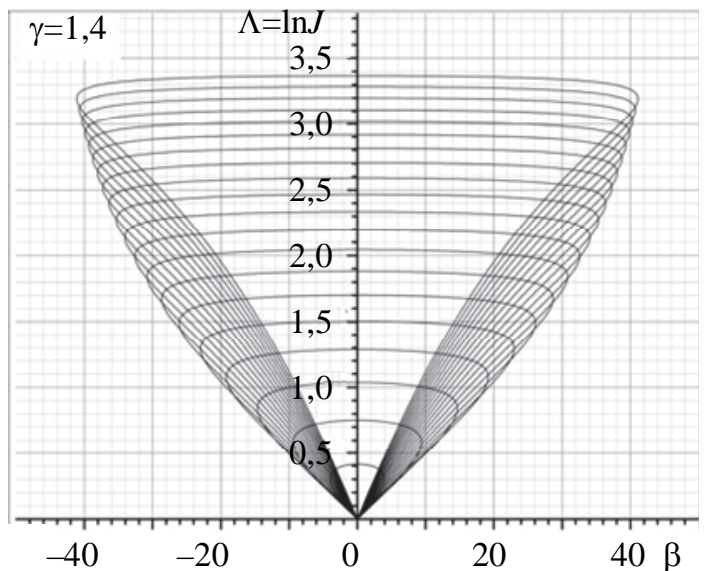

Рис. 2. Ударная поляра при $ү=1,4$, число Маха изменяется от 2 (самая маленькая поляра) до 5 (самая большая поляра) с шагом 0,2

Проведенное В.Н. Усковым [29] исследование сердцевидных кривых позволило установить их важные свойства: наличие огибающей, предельных углов отклонения потока на разрыве, точек, соответствующих разрывам, числа Маха за которыми равны единице. Можно отметить, что наличие огибающей важно в задачах сверхзвуковой аэродинамики [30], так как соответствует экстремумам давления на сторонах тела, летящего с заданным углом атаки, но с переменной скоростью. Получены результаты для оптимальных (в смысле достижения экстремума некоторого параметра) одномерных бегущих [31] и двухмерных косых ударных волн [32].

Соотношения, описывающие сердцевидные кривые, известны давно, но их использование до сих пор нередко вызывает затруднения из-за имеющихся вычислительных особенностей и необходимости отбора из множества формальных корней.

Первые количественные экспериментальные результаты, которые можно было бы сравнить с теорией, получены Вьелем в 1899 г. [33]. Он проводил измерения скорости распространения ударной волны в трубе после разрыва мембраны (прообраз современной ударной трубы). Стодола в 1903 г. [34] изучал течения внутри сопла на режиме с пусковым скачком уплотнения внутри него. Эти исследования позволили получить экспериментальное подтверждение теории Стокса, Римана, Рэнкина и Гюгонио для одиночного разрыва. Однако в УВП могут участвовать не только одиночные волны и разрывы, но и ударноволновые структуры (УВС). Ниже рассматривается история изучения УВС.

\section{Ударно-волновые структуры}

Ударно-волновые структуры и классификация образующих их разрывов

Ударно-волновые структуры возникают в результате взаимодействия (пересечения, интерференции) волн или разрывов между собой, с тангенциальными, контактными, свободными или твердыми поверхностями. По отношению к точке, в которой образуется УВС, газодинамические разрывы делятся на приходящие $\left(R_{a}\right)$ и исходящцие $\left(R_{p}\right)$, как это показано на рис. 3.

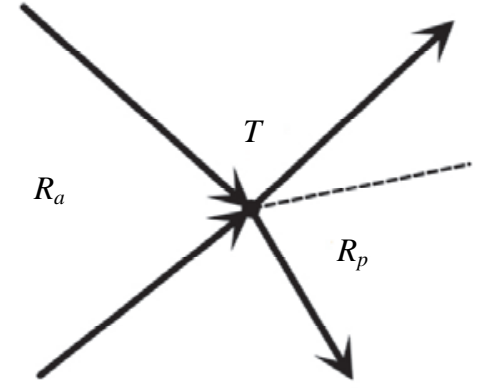

a

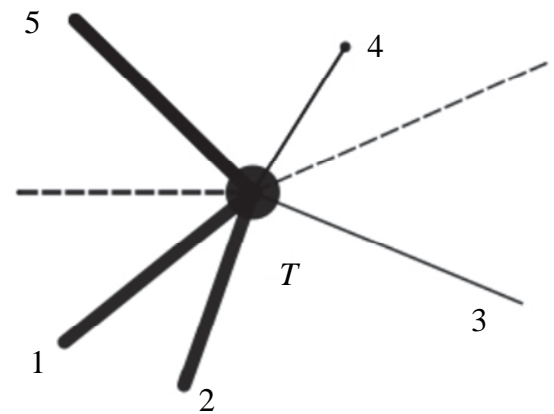

б

Рис. 3. Приходящие $R_{a}$ в точку взаимодействия $T$ и исходящие из нее $R_{p}$ разрывы (а) и обобщенная ударно-волновая структура (б): 1, 2 - приходящие скачки уплотнения одного направления; 3 - отраженный исходящий разрыв (скачок или волна); 4 - главный исходящий разрыв; 5 - приходящий встречный скачок уплотнения; - - - - тангенциальные разрывы

Причиной образования УВС являются приходящие в одну точку волны и разрывы. Исходящие волны (разрывы) представляют собой следствие взаимодействия приходящих волн (разрывов). У приходящих разрывов проекция вектора скорости на плоскость разрыва направлена к точке интерференции, а у 
исходящих - от нее (рис. 3, а).

Обобщенная УВС. Существуют разные подходы к исследованию УВС и решению задачи об интерференции ГДР. В.Н. Усковым в 1979 г. был предложен подход на основе обобщенной УВС [35]. Обобщенная УВС составлена из всех возможных типов разрывов и волн (рис. 3, б): трех приходящих 1, 2 и 5 , одного главного исходящего 4, одного тангенциального и одного отраженного разрывов 3. Последний может быть как скачком уплотнения, так и волной разрежения. Приходящие скачки уплотнения могут быть одного направления 1 и 2 или противоположных направлений 5 и 1. Последние еще называются встречными скачками, а разрывы одного направления - догоняющими. Различают образование УВС в результате взаимодействия догоняющих или встречных разрывов, распада или ветвления разрывов. Задача о расчете любой УВС сводится к определению характеристик исходящих разрывов по известным параметрам приходящих разрывов.

Формула интерференции в общем виде может быть записана как

$$
\sum R_{a}(k) \rightarrow \sum R_{p}(k) \text {. }
$$

Если в конкретной задаче нулевого порядка отсутствуют некоторые из приходящих разрывов $\left(\vec{\sigma}_{1}\right.$, $\vec{\sigma}_{2}$ или $\vec{\sigma}_{5}$ ), то интенсивность такого разрыва следует считать равной нулю. Например, взаимодействие догоняющих скачков уплотнения (разрывов одного направления) можно представить в виде

$$
\vec{\sigma}_{1}+\vec{\sigma}_{2} \rightarrow \bar{r}_{3}+\hat{\tau}+\vec{\sigma}_{4} \text {. }
$$

Тангенциальный разрыв $\tau$ разделяет два потока, прошедших через разрывы 1 и 2 (догоняющие приходящие скачки уплотнения), и отраженный 3. Для встречных скачков имеем

$$
\gamma=1,4 \vec{\sigma}_{1}+\vec{\sigma}_{5} \rightarrow \bar{\sigma}_{3}+\hat{\tau}+\vec{\sigma}_{4} .
$$

Догоняющие скачки применяются в традиционных воздухозаборниках внешнего сжатия (рис. 4), встречные - в перспективных воздухозаборниках внутреннего и смешанного сжатия. УВС могут объединяться в более сложные системы с заранее не известной структурой, состоящие из тройных конфигураций ударных волн, объединенных мостообразными скачками.

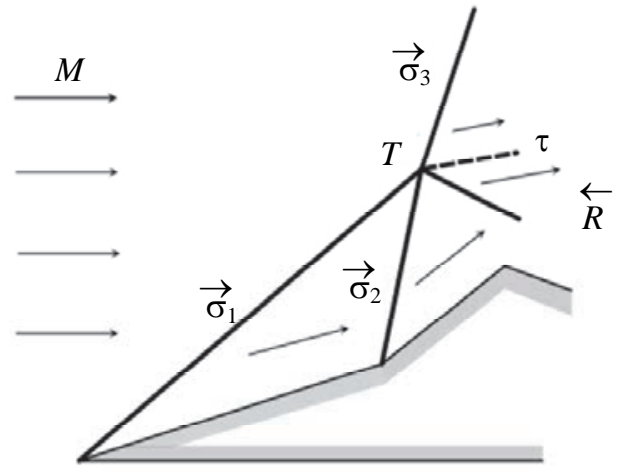

Рис. 4. Догоняющие скачки уплотнения $\sigma_{1}$ и $\sigma_{2}$ В воздухозаборнике внешнего сжатия.

$T$ - тройная точка; $R$ - отраженный разрыв; $\sigma_{i}-$ скачки уплотнения

Задачи проектирования сверхзвуковых летательных аппаратов вызвали в 40-е годы XX века активизацию исследования взаимодействий между собой отдельных волн и разрывов: волны разрежения со скачком уплотнения, ударной волны с контактным разрывом (рефракция ударной волны) и т.п. В первых экспериментах с помощью ударной трубы были изучены одномерные взаимодействия. Теория течения газа в ударной трубе в одномерной постановке предложена Шардиным в 1932 г. [36]. В Университете Торонто в 50-е годы был проведен ряд экспериментальных и теоретических работ по исследованию взаимодействия одномерных бегущих волн и разрывов:

- рефракция бегущей ударной волны на контактном разрыве - Битондо и другие [37], Битондо [38], Форд и Гласс [39];

- взаимодействие догоняющих ударных волн - Гоулд [40];

- взаимодействие ударной волны с волной разрежения - Ничолл [41] и рефракция волны разрежения Биллингтон и Гласс [42], Биллингтон [43].

Теоретические результаты были скромнее. В работе Тауба [44] исследовано распространение ударной волны по двум первоначально покоящимся газам, разделенным поверхностью раздела (контактным разрывом). Двух- и трехмерные задачи долгое время решались исключительно численными методами. Особенно актуальными являлись задачи изучения интерференции скачков уплотнения и волн разрежения при обтекании летательных аппаратов и их воздухозаборников сверхзвуковым потоком. Общность математического аппарата одномерных нестационарных и двумерных стационарных задач о взаимодействии волн и разрывов, продемонстрированная в работах В.Н. Ускова и Л.П. Архиповой $[45,46]$, позволила М.Г. Чернышову решить ряд практически важных задач [47-50] о взаимодействии косого 
скачка с волной Прандтля-Майера. Следующим шагом стало исследование взаимодействия ударной волны и прямого скачка уплотнения [51], а также косых ударных волн [52] и нестационарных тройных конфигураций [53].

Успешные численные и аналитические исследования поставили вопрос о возможности перехода от решения элементарных задач об интерференции волн и разрывов к конструированию УВС с заданными свойствами и оптимальному управлению ими.

Первой и самой важной стала задача минимизации потерь полного давления в УВС многоскачкового сверхзвукового воздухозаборника. Первым ее решил Осватич [54], который показал, что оптимальная УВС должна состоять из косых скачков одинаковой интенсивности. Замыкающий прямой скачок должен иметь немного меньшую интенсивность, но этим отличием можно пренебречь. Независимо от более ранних работ поведение функции потерь полного давления за серией косых и прямого скачков уплотнения исследовалось в докторской диссертации В.Н. Ускова. Систематический характер работы по оптимальным (экстремальным по заданному параметру) УВС приняли только в 90-е годы, после выхода постановочной статьи В.Н. Ускова и А.В. Омельченко [55] и двух работ А.В. Омельченко [56, 57], в которых к изучению УВС была привлечена теория оптимального управления. В течение пяти лет, начиная с 1995 г., В.Н. Усковым и А.В. Омельченко были исследованы на экстремум основные зависимости газодинамических переменных за УВС для следующих практически важных случаев:

- одномерные бегущие волны [28];

- торможение сверхзвукового потока в диффузоре [58];

- экстремумы за оптимальными УВС с учетом того, что угол разворота потока не должен превышать предельное значение для угла разворота на скачке [59];

- УВС с максимально возможным углом отклонения потока [60];

- оптимальные системы догоняющих скачков уплотнения в многоскачковых воздухозаборниках [61];

- оптимальная система, составленная из скачка уплотнения и волны разрежения [62].

Решение общих задач позволило М.Г. Чернышову и В.Н. Ускову перейти к решению задач оптимального управления струйными течениями [63] и внешним обтеканием летательного аппарата сверхзвуковым потоком [30, 64-66].

\section{Тройные конфигурации ударных волн}

Теория тройных конфигураций (ТК) ударных волн имеет огромное значение для проектирования современных воздухозаборников, рассчитанных на большие сверхзвуковые скорости полета, а также детонационных двигателей. Как будет показано дальше, ТК тесно связаны с понятием регулярной и нерегулярной интерференции ГДР. Тройными конфигурациями ударных волн называются УВС, состоящие из одного приходящего скачка, одного основного скачка и третьего скачка, который может быть как приходящим, так и отраженным (рис. 5). Из тройной точки, в которой сходятся все три скачка, всегда исходит тангенциальный разрыв $\tau$.
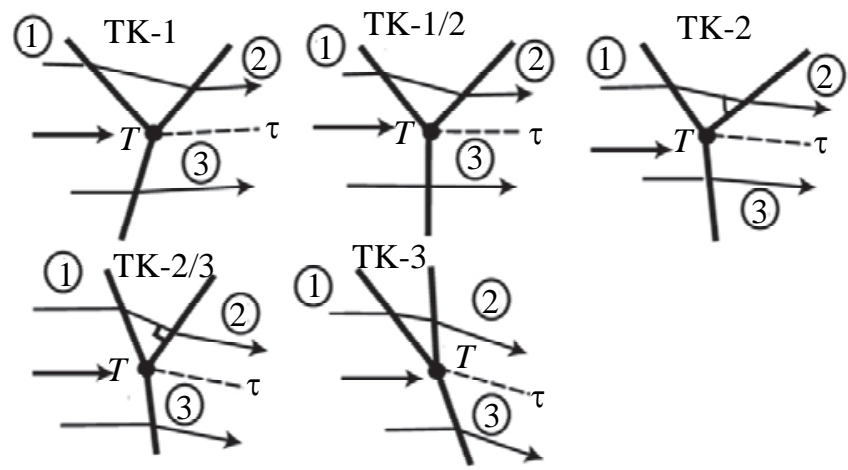

Рис. 5. Тройные конфигурации ударных волн: 1 - ветвящийся скачок; 2 - второй скачок; 3 - главный скачок

В.Н. Усковым введено разделение ТК на конфигурации трех основных типов в зависимости от направлений отклонения потока на скачках - ТК-1, ТК-2, ТК-3 [67], и двух переходных - ТК-1/2 и ТК-2/3. В том случае, когда первый (ветвящийся) и главный (3 на рис. 5) скачки в окрестности тройной точки отклоняют поток в разных направлениях, тройную конфигурацию относят к первому типу (ТК-1). Тройная конфигурация с прямым главным скачком называется стационарной маховской конфигурацией (СМК или ТК-1/2) и является пограничной между первым и вторым типами. В тройной конфигурации второго типа (ТК-2) первый и главный скачки отклоняют поток в одном, а скачок 2 - в противоположном направлении. Конфигурация с прямым (нормальным к вектору скорости потока перед ним) скачком 2 является переходной от второго типа к третьему и обозначается ТК-2/3. В конфигурации третьего типа 
(ТК-3) все скачки отклоняют поток в одном направлении. В.Н. Усковым также была решена задача о расчете параметров тройных конфигураций стационарных скачков уплотнения [68].

Как будет показано ниже, ТК-1 имеет отношение к нерегулярной интерференции встречных скачков, и ТК-1 применяются в воздухозаборниках внутреннего сжатия; ТК-2 - к отражению скачка уплотнения от стенки, они применяются в воздухозаборниках смешанного типа с внутренним переотражением скачков от стенки. Кроме того, ТК-2 образуются в осесимметричных сверхзвуковых струях при отражении висячего скачка уплотнения от оси с образованием диска Маха. На невозможность регулярного отражения скачка уплотнения от оси симметрии без образования диска Маха было впервые указано еще в работе Д.А. Мельникова [69], доказательство было позднее уточнено в работах [70, 71]. Действительно, на оси симметрии должны выполняться условия равенства нулю угла наклона вектора скорости и кривизны линии тока за отраженным скачком, но при $y=0$ это невозможно. При приближении падающего скачка к оси симметрии его кривизна $K_{\sigma}$ стремится к бесконечности, так как $K_{\sigma} \sim y^{-1}$, поэтому всегда создаются условия образования маховского диска в нерасчетной струе. ТК-3 - это частный случай взаимодействия догоняющих скачков, когда отраженный разрыв вырождается в характеристику, в других случаях он является или скачком, или волной разрежения. Интерференция догоняющих скачков здесь далее не рассматривается.

Теоретическое исследование тройной конфигурации ударных волн было впервые выполнено фон Нейманом [72]. Именно фон Нейман предположил, что из тройной точки исходит тангенциальный разрыв (поверхность скольжения), разделяющий потоки за отраженной и за главной ударными волнами. Курант и Фридрихс [73] показали, что в рамках модели идеального газа три ударные волны могут существовать в одной точке только при наличии еще какой-либо поверхности разрыва.

В работе Бликни, Флэтчера и Вэймера [74] и других экспериментально проверялось предположение фон Неймана о существовании тангенциального разрыва за тройной точкой. Во многих экспериментах контактный разрыв наблюдался, а в тех случаях, когда он не наблюдался, теоретическое значение перепада плотностей по его сторонам не превосходило погрешности измерения, поэтому его и не удавалось зафиксировать. В 1967 г. Брид развил результат Куранта и Фридрихса на случай произвольных уравнений состояния газа [75]. Таким образом, модель фон Неймана была доказана.

Помимо модели фон Неймана, было предложено несколько других возможных локальных картин течения в тройной точке. В 1959 г. Стернберг [76] высказал предположение, что в ближайшей окрестности тройной точки поверхность тангенциального разрыва не является полностью сформировавшейся. Стернберг произвел расчет течения в окрестности тройной точки ТК с учетом вязкости газа.

Описание тройной точки вообще без использования УДС на ГДР, т.е. без использования соотношений Рэнкина-Гюгонио, было впервые предпринято в 1964 г. в работе Сакураи [77]. Он получил приближенное аналитическое решение уравнений Навье-Стокса в малой окрестности тройной точки. Сакураи нашел газодинамические параметры в окрестности тройной точки в зависимости от полярного угла и показал, что при очень малых значениях интенсивности скачков полученная им теория лучше соответствует эксперименту, чем теория фон Неймана. При больших интенсивностях скачков, напротив, теория фон Неймана более точна. Такой результат представляется естественным. В малой окрестности точки интерференции сильно влияние реальных свойств газа, а ударные волны нельзя считать бесконечно тонкими. По мере удаления от точки интерференции снижается точность разложения уравнений в ряд по малому параметру.

Стоит упомянуть и вовсе экзотические гипотезы. В работе В.Г. Дулова [78] и в работе В.Г. Дулова и Г.А. Лукьянова [79] было высказано предположение, что из тройной точки выходит не один, а два тангенциальных разрыва, однако это предположение не нашло экспериментального подтверждения и не получило математического развития. В настоящее время модель фон Неймана считается общепризнанной.

В результате исследования общего уравнения интерференции (14) В.Н. Усковым и М.В. Чернышовым были определены области существования (рис. 6) ТК различных типов [80], а также УВС, содержащих догоняющие скачки уплотнения одного направления [81].

И наконец, в 1990 г. А.Л. Старых было выполнено комплексное исследование областей существования различных решений общего уравнения интерференции ГДР [82], а методика расчета параметров тройных конфигураций была доведена до пакета прикладных программ [83]. Следующее десятилетие прошло под знаком изучения экстремальных свойств тройных конфигураций ударных волн, как стационарных, так и нестационарных. В 2006 г. вышли в свет две постановочные работы В.Н. Ускова и М.Г. Чернышова по оптимальным УВС [84] и экстремальным задачам управления струйными течениями [85]. Другая группа работ была посвящена оптимальным тройным конфигурациям, сначала стационарным маховским [86], а затем и всем остальным: для стационарного [80, 84] и нестационарного случаев [87].

Таким образом, под руководством В.Н. Ускова с 1995 г. было выполнено комплексное исследование экстремальных свойств тройных конфигураций ударных волн. В.Н. Усковым совместно с М.В.Чернышевым в 2008 г. было представлено полное исследование стационарных ТК, отвечающих модели фон Неймана, позднее обобщенное на нестационарный случай [88]. 


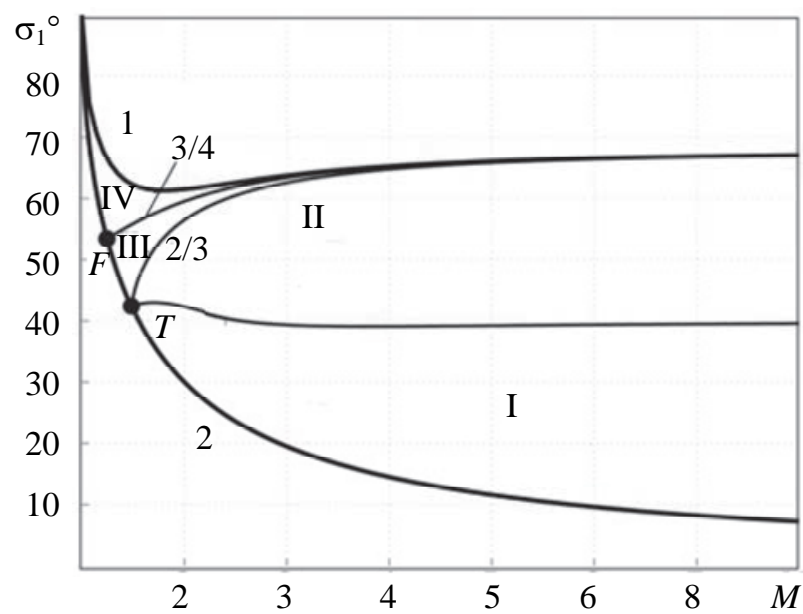

Рис. 6. Области существования тройных конфигураций: I - TК-1; II - TK-2; III - TK-3; IV - область, где тройные конфигурации существовать не могут. $F$ - число Маха $M_{F}=1,245, T$ - число Маха $M_{T}=1,483$ для $Y=1,4, \sigma-$ угол наклона скачка уплотнения в градусах

Модель фон Неймана для реального газа рассмотрена Ло для кислорода [89] и Бен-Дором [90] для азота и аргона. В этих работах калорические свойства газов описывались с помощью набора колебательных уровней энергии молекул, для каждого из которых была задана энергия его активации и его кратность. В работе Ли и Гласса [91] утверждается, что при расчете ТК в кислороде $\mathrm{O}_{2}$ и азоте $\mathrm{N}_{2}$ можно пользоваться моделью идеального газа, а при расчете в углекислом газе $\mathrm{CO}_{2}$ и шестифтористой сере $\mathrm{SF}_{6}$ необходимо использовать уже модель несовершенного газа. Сравнение различных моделей двухатомного несовершенного газа в приложении к расчету ТК было выполнено В.Н. Усковым и П.С. Мостовых [92].

\section{Встречные ударные волны и отражение ударной волны от стенки}

История изучения набегания ударной волны на клин

Первые УВС были описаны Махом [93]. Он экспериментально зафиксировал бегущие ударные волны и возникающие из них УВС, полученные при закорачивании электрических конденсаторов с образованием искры. В своей работе он описал два вида отражения бегущей ударной волны от наклонной поверхности (рис. 7):

- регулярное отражение, которое состоит из двух ударных волн - падающей волны, приходящей на твердую поверхность, и отраженной, исходящей из точки падения (рис. 7, б);

- нерегулярное отражеене, которое состоит из трех ударных волн - падающей, отраженной и главной, имеющих общую точку; такой вид отражения носит название простого маховского, а соответствующая конфигурация, если она не содержит других нормальных разрывов, - тройной конфигурации ударных волн (рис. 7, а).

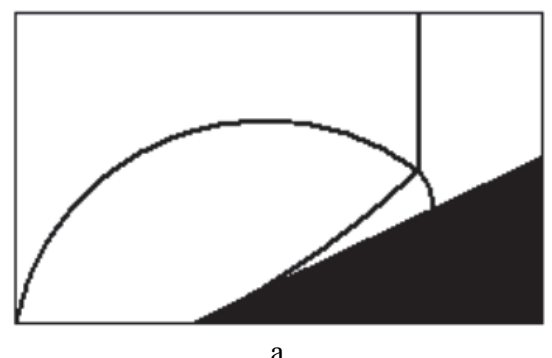

a

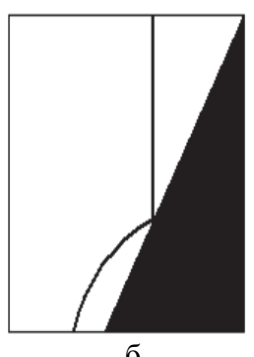

6

Рис. 7. Нерегулярное маховское (а) и регулярное (б) отражение ударной волны от наклонной стенки в опытах Э. Maха (1878 г.)

Нейман сформулировал двух- и трехволновые теории отражения ударной волны от клина. Он же высказал идею о том, что отражение может быть правильным (регулярным) и неправильным (нерегулярным, как альтернатива правильному), а маховское отражение есть одно из возможных типов нерегулярного отражения. Нейман также качественно верно описал вид нерегулярного отражения (рис. 8), который он назвал немаховским и который впоследствии получил название «отражение Неймана» [94]. Экспериментальные исследования взаимодействия бегущей ударной волны с неподвижным клином, выполненные Смитом в 1945 г. [95], показали зависимость характера отражения волны от угла при вершине клина. Смит установил также, что при больших скоростях падающей ударной волны на отраженной ударной волне в маховском отражении образуется излом. Такую УВС Смит назвал сложным маховским отраже- 
нием (рис. 9, а). В 1951 г. Уайт [96] обнаружил, что при еще больших скоростях падающей ударной волны отраженная ударная волна сама выступает как падающая и образует вторую тройную конфигурацию. Данную УВС называют двойным маховским отражением или отражением Маха-Уайта (рис. 9, б).

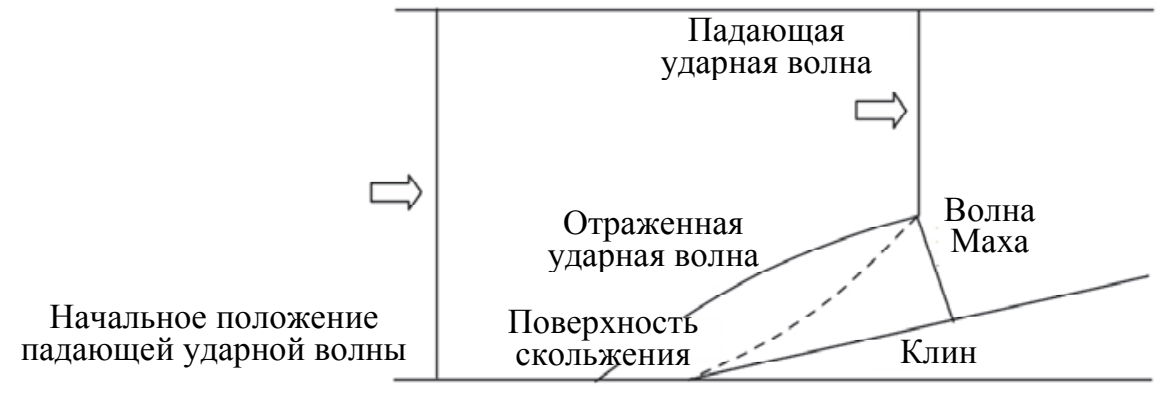

Рис. 8. Схема нерегулярного (маховского) отражения ударной волны от клина

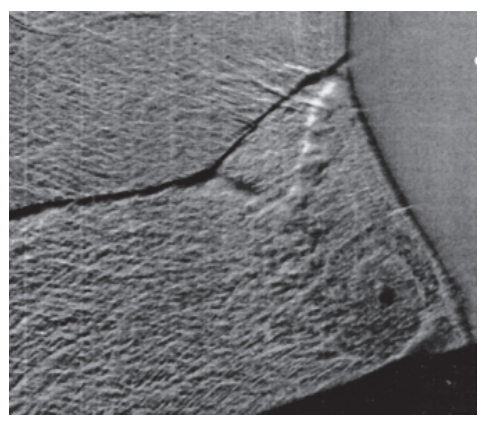

a

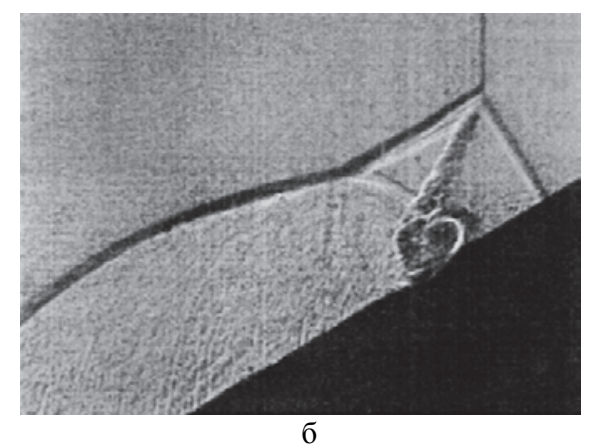

Рис. 9. Сложное (а) и двойное (б) маховское отражение

По мере распространения ударной волны по клину тройная точка может оставаться на постоянном расстоянии от поверхности клина (стационарное маховское отражение), удаляться от нее (прямое маховское отражение) или приближаться к ней (инверсное маховское отражение). Таким образом, в зависимости от угла клина и скорости волны возможны существования весьма разнообразных ударно-волновых конфигураций. Их классификация и наиболее полный обзор приведен в труде Бен-Дора [97].

\section{Отражение слабых ударных волн от клина - парадокс Неймана}

При числах Маха $M<M_{T}$ (для воздуха $M_{T}=1,483$, точка $T$ на рис. 6) тройные конфигурации ТК-2 существовать не могут. Соответственно, не может существовать маховское отражение скачка от стенки или плоскости симметрии. Существуют предположения, что при $M=M_{T}$ происходит переход от ТК-2 к ТК-3, т.е. второй скачок из исходящего становится приходящим. Каков механизм такой перестройки, пока не выяснено. Область существования ТК-3 ограничена числом Маха $M_{F}=1,245$ (точка $F$ на рис. 6 ). При числах $M<M_{F}$, тройные конфигурации ударных волн ТК-3, соответствующие взаимодействию догоняющих скачков уплотнения одного направления, существовать не могут. Обязательным является наличие отраженного разрыва - скачка или волны разрежения (область IV на рис. 6). Первым на это обратил внимание фон Нейман: теоретический анализ показывал, что маховское отражение существовать при малых числах Маха не может, но в экспериментах нечто подобное наблюдалось. Данный факт получил название парадокса Неймана. Малые числа Маха являются важнейшей областью параметров, характерной для возникновения волнового кризиса на верхней части профиля крыла, а также при истечении струй из сопел современных турбореактивных двигателей, поэтому проблеме парадокса Неймана было уделено большое внимание.

Подробный обзор состояния этого вопроса приведен в 1949 г. в работе Бликни [98]. Решению данной проблемы посвящены работы Баргманна [99], Лайтхилла [100], Тинга и Людлоффа [101], в которых использовались приближенные аналитические методы расчета полей газодинамических параметров в рассматриваемом течении. В этих работах угол при вершине клина рассматривается как малый параметр, по которому проводится разложение решения в ряд. В качестве нулевого приближения рассматривается течение, реализующееся при нулевом угле при вершине клина. Флэтчер и др. [102] в 1951 г. опубликовали экспериментальные данные для клина с углом при вершине $10^{\circ}$. Сравнение с выполненными ранее расчетами показало, что теоретически полученные результаты качественно хорошо повторяют эксперимент, но количественно заметно отличаются от него.

На протяжении почти сорока лет проводились эксперименты [103-105], порой весьма тонкие, которые однозначно продемонстрировали, что трехволновая теория фон Неймана не работает [106] при отражении слабых ударных волн с числом Маха набегающего потока меньше особого числа Маха $M=M_{T}$. 
При этих числах Маха вторичная ударная поляра не пересекается с основной полярой при любых значениях интенсивности приходящего скачка, полностью располагаясь внутри нее (о методе анализа ударных поляр речь пойдет ниже). В.Н. Усковым было определено точное значение $M_{T}=\sqrt{(2-\varepsilon) /(1-\varepsilon)}, \varepsilon=(\gamma-1) /(\gamma+1)$.

Гудерлей, применив к случаю слабых ударных волн (малых числах Маха) метод годографа скорости [107], предположил, что модель тройной конфигурации фон Неймана в данном случае не работает, и предложил в 1964 г. четырехволновую модель (рис. 10, а) с дополнительной слабой волной разрежения за отраженным разрывом [108].

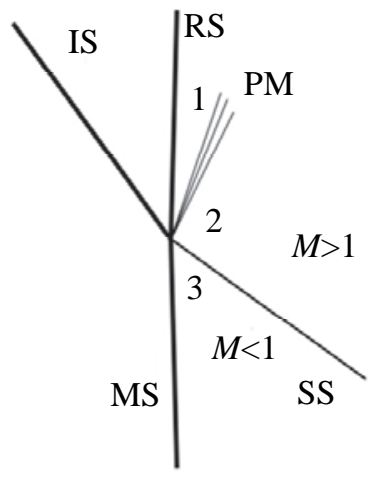

a

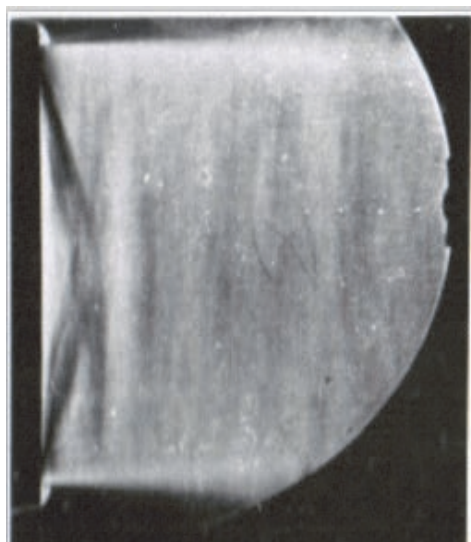

б

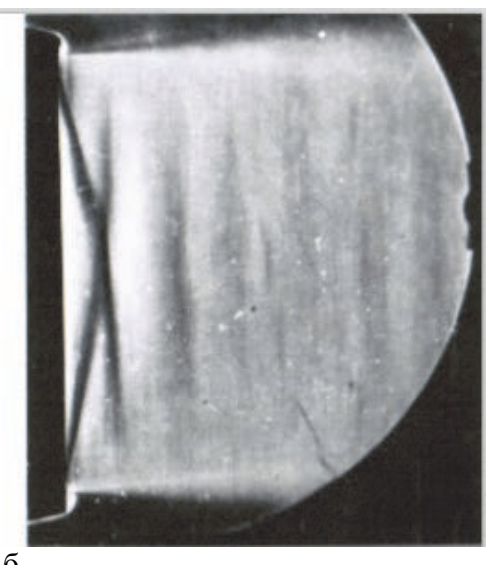

Рис. 10. Четырехволновая модель Гудерлея (а) и соответствующая картина истечения струи из сопла при малых числах Маха (б), диаметр сопла - 50 мм: IS - падающий скачок; RS - отраженный скачок; MS - ножка Маха; PM - волна разрежения Прандтля-Майера; SS - тангенциальный разрыв

Модель Гудерлея исследовалась в 1999 г. в работе Е.И. Васильева и А.Н. Крайко [109]. Долгое время не удавалось получить численное решение для такого рода потоков, пока Е.И. Васильев [110] не продемонстрировал, что все дело в недостаточной точности численных методов, влиянии «схемной» вычислительной вязкости и паразитных осцилляций решения, а течение отвечает «четырехволновой» модели Гудерлея. Для этого был использован численный метод с выделением разрывов [111]. В результате сегодня такая УВС получила название Гудерлея-Васильева. И наконец, В.Н. Усков, построив стройную классификацию видов интерференции стационарных ГДР, показал, что тройные конфигурации бывают трех типов, а модель Гудерлея - это всего лишь частный случай интерференции догоняющих скачков уплотнения [112]. Кроме того, модель Гудерлея, вообе говоря, неправильная, так как скачок, названный Гудерлеем отраженным (RS на рис. 10, a), на самом деле является вторым приходящим скачком (2 на рис. 3, б).

\section{Регулярная (РИ) и маховская интерференция (МИ) встречных ударных волн}

Буземан в ряде работ в 1929-1937 г.г. [113-115] заложил основу графических методов решения задач об интерференции ГДР с помощью ударных поляр, связывающих интенсивность косых скачков уплотнения с углом разворота потока на скачке. Ударные поляры именно с того времени называются в его честь полярами Буземана. За характерный вид их еще называют сердцевидными кривыми. Еще одно название - изомахи, так как каждая ударная поляра строится для конкретного числа Маха набегающего потока. Методы решения задач интерференции разрывов с помощью ударных поляр были развиты Курантом и Фридрихсом в 1947 г. в работе [73] и усовершенствованы в 1956 г. Кавамура и Саито [116], которые впервые применили их к анализу отражения ударной волны от клина. В некотором смысле задача изучения интерференции встречных скачков является обобщением случая отражения косого скачка от стенки или от плоскости симметрии [117], но взаимодействующие скачки (ударные волны) могут иметь разные интенсивности, в результате картины течения отличаются бо́льшим разнообразием, чем в случае отражения волны от стенки.

Проще всего представить встречные ударные волны в одномерном случае (рис. 11, а), в большей размерности этому соответствует РИ косых скачков уплотнения (рис. 11, б).

В 1960 г. Молдер [118] разработал аналитическую теорию регулярного взаимодействия встречных ударных волн, опираясь на графический метод анализа ударных поляр (рис. 11, в). Основные соотношения для анализа РИ встречных скачков приведены в работе [119]. Если точка пересечения лежит внутри главной поляры (рис. 1, в), то эти условия являются необходимыми и достаточными для существования РИ. Если поляры не пересекаются внутри главной поляры, то реализуется МИ (рис. 12). При некотором 
сочетании параметров поляры могут пересекаться вне главной поляры. Это означает, что возможна как РИ, так и МИ. Этот случай будет рассмотрен ниже отдельно.

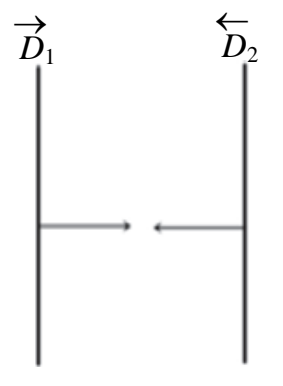

a

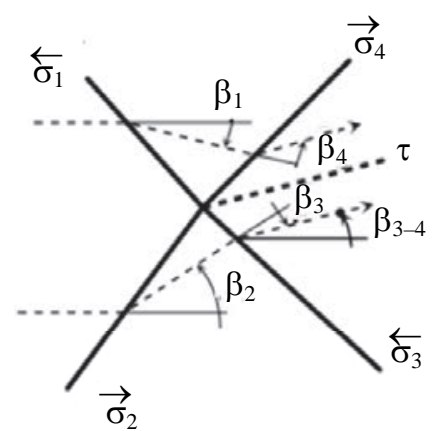

6

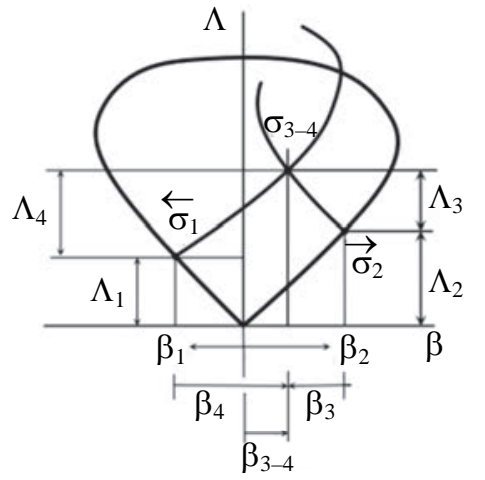

B

Рис. 11. Регулярная интерференция встречных ударных волн $D_{1}$ и $D_{2}($ a), косых скачков уплотнения (б) и соответствующее решение на плоскости поляр (в). $\beta_{i}-$ углы разворота потока на соответствующем $i$-ом скачке; $\bigwedge_{i}$ - логарифм интенсивности соответствующего $i$-го скачка

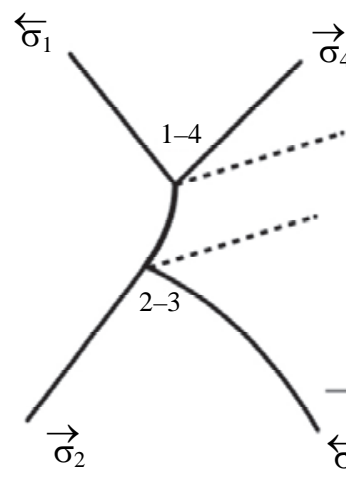

a

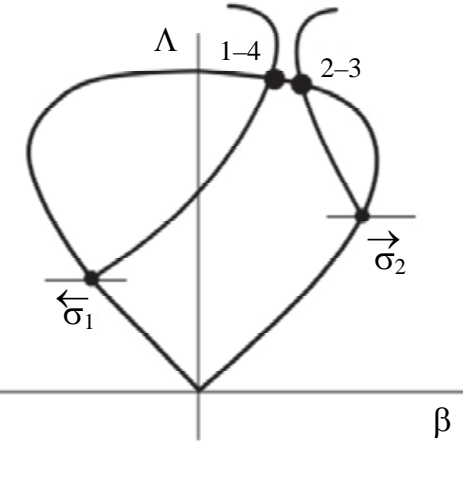

6

Рис. 12. Маховская интерференция встречных ударных волн (а) и соответствующее решение на плоскости поляр (б)

На рис. 11, в, приведено графическое решение для случая регулярного несимметричного взаимодействия встречных скачков $\sigma_{1}$ и $\sigma_{2}$, разворачивающие исходное течение в противоположном направлении на углы $\beta_{1}$ и $\beta_{2}$. За скачками $\sigma_{3}$ и $\sigma_{4}$ потоки должны быть параллельны друг другу, поэтому скачок $\sigma_{4}$ разворачивает поток в направлении, противоположном развороту потоку на скачке $\sigma_{1}$. Со скачками $\sigma_{2}$ и $\sigma_{3}$ дело обстоит аналогично. Итоговый угол разворота $\beta_{3-4}$ определяется как алгебраическая сумма углов разворота потока на всех скачках, что показано на рис. 11, в. Давление за скачками $\sigma_{3}$ и $\sigma_{4}$ должны быть равны, поэтому $\Lambda_{1}+\Lambda_{4}=\Lambda_{2}+\Lambda_{3}$ (рис. 11 , в).

Очевидно, что при равенстве интенсивности скачков $\sigma_{1}$ и $\sigma_{2}$, итоговый угол разворота потока $\beta_{3-4}$ будет равен нулю, картина на плоскости поляр полностью симметрична, и в данном случае взаимодействие встречных скачков аналогично случаю регулярного отражения скачка от стенки [120].

Если при РИ зафиксировать интенсивность одного из скачков, например, $\sigma_{1}$, а интенсивность второго скачка $\sigma_{2}$ увеличивать, то увеличивается интенсивность накрест лежащих скачков (в рассматриваемом случае $\left.\sigma_{3}\right)$, а интенсивность прилежащего скачка $\sigma_{4}$ уменьшается. Зависимость интенсивности отраженных скачков $\sigma_{3}$ и $\sigma_{4}$ от интенсивности взаимодействующих скачков $\sigma_{1}$ и $\sigma_{2}$ приведена на рис. 13. Как видно, интенсивности накрест лежащих скачков уплотнения могут отличаться весьма значительно, что весьма перспективно для организации стационарной детонации в гиперзвуковых воздушно-реактивных двигателях.

На рис. 14 приведена зависимость интенсивности отраженного скачка от интенсивности приходящих скачков при регулярной и нерегулярной интерференции (рис. 14, б) в случаях, когда переход РИ↔МИ осуществляется в соответствии с критерием СМК (рис. 14, а) и в соответствии с критерием фон Неймана (рис. 14, б). Видно, что в последнем случае переход РИ↔МИ сопровождается скачкообразным изменением интенсивности отраженного разрыва. При переходе в соответствии с критерием СМК интенсивность отраженного разрыва изменяется плавно. 


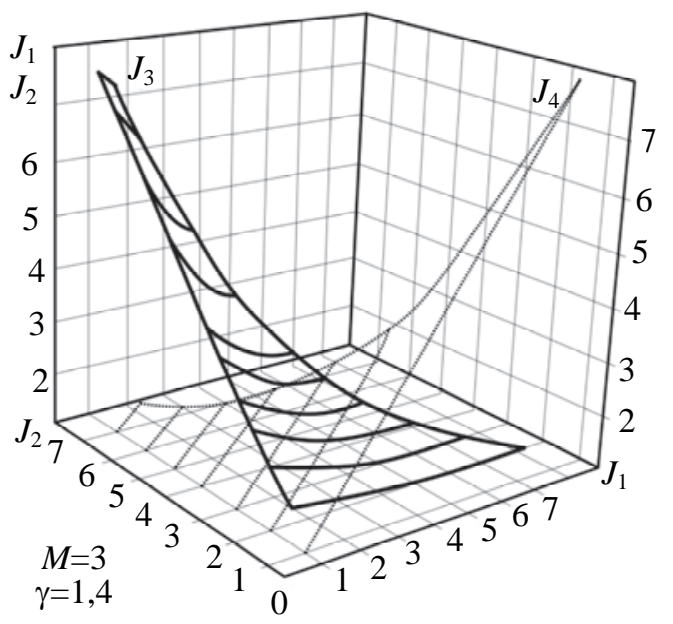

Рис. 13. Зависимость интенсивности отраженных скачков от интенсивности приходящих скачков при регулярной интерференции встречных скачков

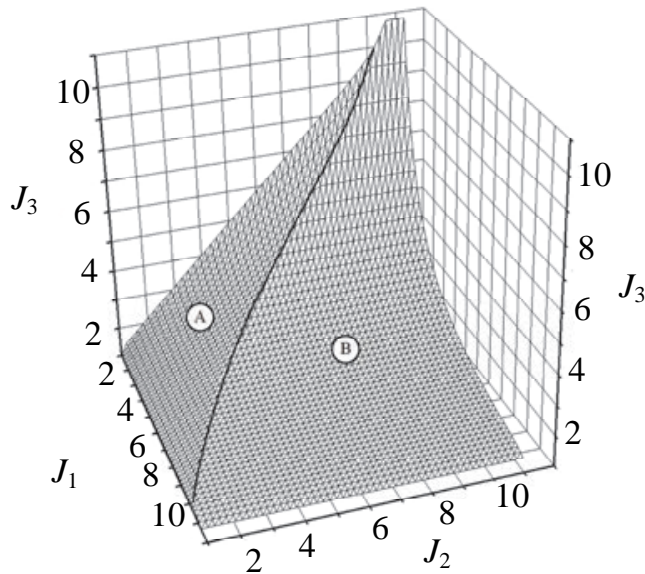

a

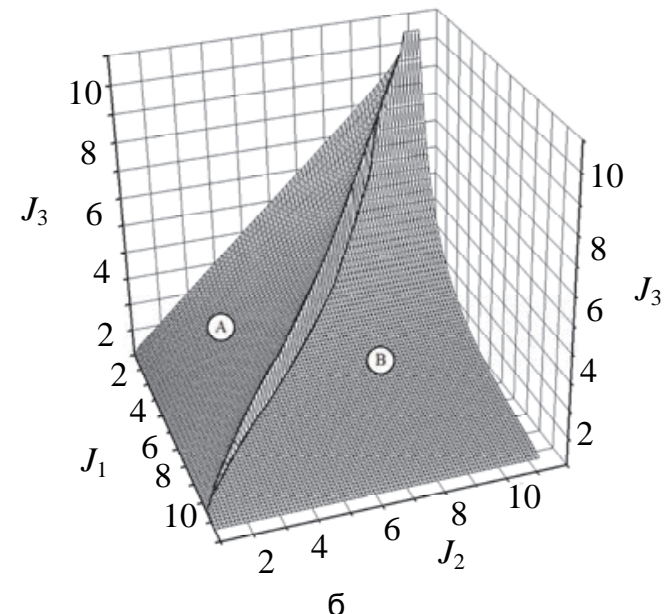

Рис. 14. Зависимость интенсивности отраженного скачка от интенсивности приходящих скачков в случае, когда переход РИ↔МИ осуществляется в соответствии с критерием стационарной маховской конфигурации (а) и в соответствии с критерием фон Неймана (б): а - регулярная интерференция; б - маховская интерференция. $M=3,5$

\section{Критерии перехода между маховской и регулярной интерференцией}

Для случая отражения косой ударной волны от стенки фон Нейман сформулировал «критерий отсоединения» (criterion detaching), в соответствии с которым переход от регулярного отражения к маховскому происходит в момент, когда вторичная поляра касается оси ординат (рис. 15, а, г). В его честь данный критерий называется критерием фон Неймана. Соответствующую интенсивность приходящего скачка принято обозначать $\Lambda_{R}=\ln J_{R}$ :

$$
\begin{aligned}
A_{0} & =-(1-\varepsilon)^{2} L^{4}, L=\left(J_{R}-1\right) /\left(J_{R}+\varepsilon\right), \\
\sum_{n=0}^{3} A_{n} x_{R}^{n}=0, x_{R}=(1+\varepsilon) M^{2} /\left(J_{R}+\varepsilon\right), & =2(1-\varepsilon)(3-\varepsilon) L^{2}-4(1-\varepsilon)(1-3 \varepsilon) L^{3}+(1-\varepsilon)^{4} L^{4}, \\
A_{2} & =2 L^{2}\left(1-2 \varepsilon-\varepsilon^{2}\right)-4 L-1, \\
A_{3} & =1 .
\end{aligned}
$$

Позднее фон Нейман обратил внимание на тот факт, что в некотором диапазоне углов клина и чисел Маха ударная поляра пересекается как с осью ординат вне основной поляры (рис. 15, а), так и с верхней ветвью основной поляры. Следовательно, в данном случае теоретически возможны как регулярное, так и маховское отражение, т.е. существует область неоднозначности решения. В результате фон Нейман предложил другой критерий перехода от регулярного отражения к маховскому, несколько неудачно назвав его «критерием механического равновесия» (mechanical equilibrium). В соответствии с этим критерием переход должен происходить в момент пересечения ударной поляры с изомахой в ее вершине (рис. 15, б), т.е. интенсивность ножки Маха в данном случае равняется максимальной интенсивности для 
заданного числа Маха, определяющего изомаху, чему соответствует характерная интенсивность $J_{0}$ (или $\left.\Lambda_{0}=\ln J_{0}\right)$ :

$$
\begin{aligned}
A_{3} & =1-\varepsilon^{2}, \\
A_{2} & =-\left(\left(1+\varepsilon-\varepsilon^{2}+\varepsilon^{3}\right) J_{m}+1+\varepsilon^{2}\right), \\
\sum_{k=0}^{3} A_{k} J_{0}^{k}=0, & A_{1}=\varepsilon\left(1+J_{m}\right)\left[(1-\varepsilon) J_{m}-2\right], \\
A_{0} & =(1-\varepsilon) J_{m}\left(J_{m}-1\right), \\
J_{m} & =(1+\varepsilon) M^{2}-\varepsilon .
\end{aligned}
$$

Возникающая при этом УВС называется стационарной маховской конфигурацией (ТК-1/2 по классификации В.Н. Ускова). Именно поэтому данный критерий был назван В.Н. Усковым критерием $\boldsymbol{C M K}$. Наибольший вклад в определение областей существования различных регулярных и нерегулярных взаимодействий встречных скачков принадлежит В.Н. Ускову и А.Л. Старых [121]. Очевидно, что существует некоторое особое число Маха $M_{0 R}$, такое, что вторичная поляра, выпущенная из точки 1 основной поляры, касается оси ординат в точности в вершине основной поляры (рис. 15, в). Этому случаю соответствует уравнение

$$
\frac{4 A_{1}\left(\frac{A_{1}}{A_{0}}-3 \frac{A_{2}}{A_{1}}\right)^{2}}{9-\frac{A_{1} A_{2}}{A_{0}}}=9-\frac{A_{1} A_{2}}{A_{0}}-4\left(3-\frac{A_{2}^{2}}{A_{1}}\right),
$$

коэффициенты $A_{k}-$ те же, что и в (15). При числах Маха $M<M_{0 R}$, вторичная поляра касается оси ординат внутри основной поляры (рис. 15, г), поэтому переход от регулярного отражения к нерегулярному происходит скачком к точке пересечения левой ветви вторичной поляры с дозвуковой областью правой ветви основной поляры; переход в соответствии с критерием СМК невозможен, и интенсивность $J_{0}$ здесь не определена.

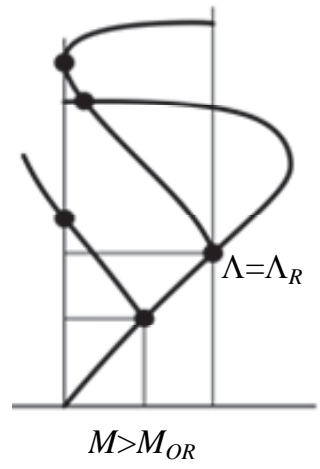

a

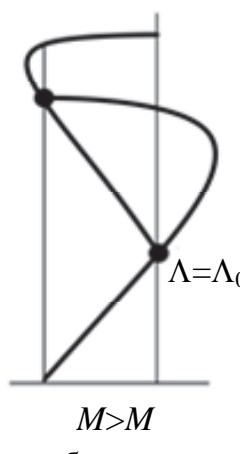

6

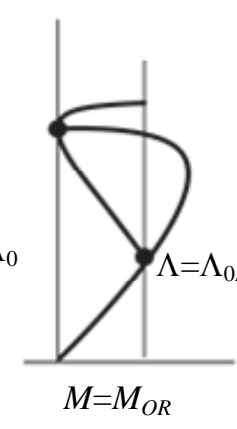

B

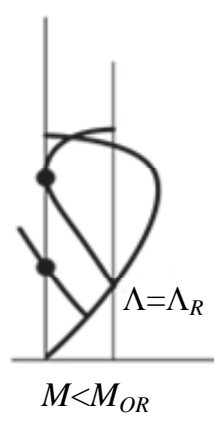

$\Gamma$

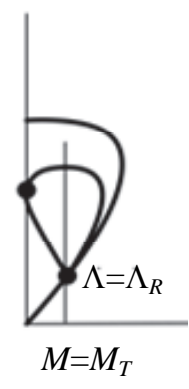

д

Рис. 15. Переход от регулярного отражения к маховскому: переход в соответствии с критерием фон Неймана при больших числах Маха (a); переход в соответствии с критерием стационарной маховской конфиигурации (б); случай особого числа Маха $M=M_{0 R}$, при котором оба критерия совпадают (в); переход

в соответствии с критерием фон Неймана при небольших числах Маха (г); предельное число Маха

$M=M_{T}$, ограничивающее область существования тройных конфигураций ударных волн (д).

$\Lambda_{R}$ - особая интенсивность приходящего скачка, при которой происходит переход к маховской

интерференции в соответствии с критерием фон Неймана; $\Lambda_{0}$ - особая интенсивность приходящего

скачка, при которой происходит переход к маховской интерференции в соответствии с критерием стационарной маховской конфигурации; $\Lambda_{O R}=\Lambda_{R}=\Lambda_{0}$ - особая интенсивность при $M=M_{0 R}$

При числах Маха $M>M_{0 R}$ вторичная ударная поляра сначала пересекает первичную поляру в ее вершине (рис. 15, б), а уже потом отсоединяется от оси ординат (самая верхняя точка на рис. 15, а). Соответственно, теоретически возможны два варианта перехода к нерегулярному отражению: в соответствии с критерием СМК, при интенсивности падающего скачка, равной $J_{0}(16)$, переход плавный; в соответствии с критерием отсоединения, при интенсивности падающего скачка, равной $J_{R}(15)$, переход происходит скачком на точку пересечения основной и вторичной поляры.

В более общем случае интерференции несимметричных встречных скачков ситуация сложнее, и формулы (15)-(17) не действуют. Границей, определяющей переход РИ↔МИ в соответствии с критерием фон Неймана, является случай, когда вторичные поляры касаются друг друга вне основной поляры 
(черная точка на рис. 16). Границей, соответствующей критерию СМК, является случай, когда точка пересечения поляр 1 и 2 лежит на основной поляре (серые точки на рис. 16 сливаются в одну). Аналогом этому при отражении скачка от стенки или оси симметрии является пересечение вторичной поляры с основной полярой в ее вершине. Для случая $J_{1} \neq J_{2}$, каждому значению $J_{2}$ соответствует своя величина $J_{01}$, удовлетворяющая критерию СМК.

Определение этой величины производится в следующей последовательности: по заданным значениям $M$ и $J_{2}$ рассчитывается тройная конфигурация ударных волн (ТК-1 или ТК-2) и находится интенсивность $J_{1-4}$ главного скачка уплотнения в этой конфигурации. Величина $J_{1-4}$ позволяет путем расчета тройной конфигурации при скачке $\sigma_{1}$ найти интенсивность $J_{01}$ другого скачка уплотнения, при которой существует данная тройная конфигурация. При этом считается, что главный скачок прямолинейный, и $J_{1-4}=J_{2-3}$. Аналогично находится $J_{R 1}$. Некоторые результаты расчетов зависимости $J_{02}\left(J_{1}\right)$ или, что то же самое, $J_{01}\left(J_{2}\right)$ для $\gamma=1,4$ и различных чисел Маха приведены на рис. 17. Прямая линия соответствует симметричному случаю $J_{1}=J_{2}$.

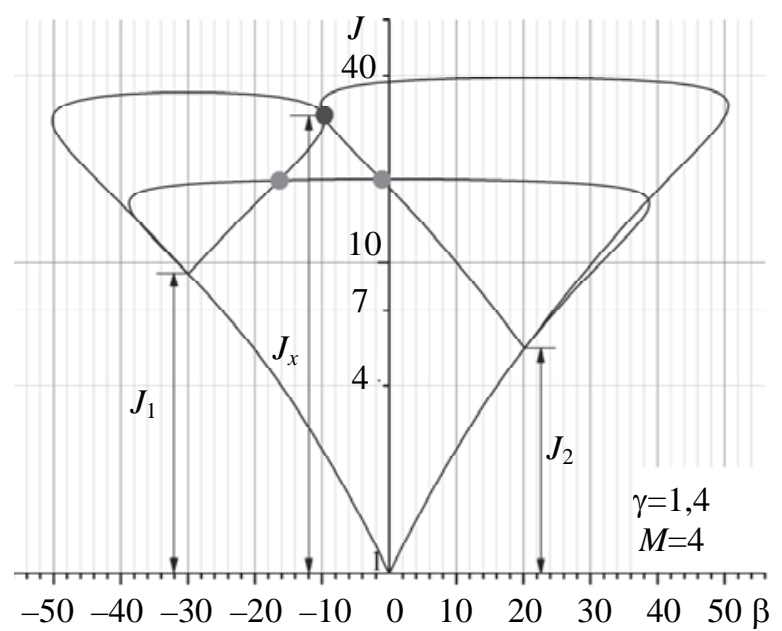

Рис. 16. Переход РИ↔МИ в соответствии с критерием фон Неймана

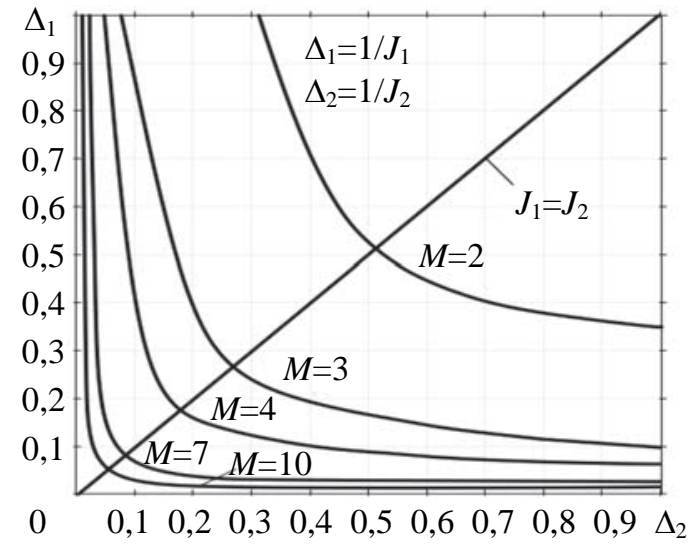

Рис. 17. Зависимость интенсивности приходящего скачка уплотнения, соответствующей началу маховской интерференции в соответствии с критерием стационарной маховской конфригурации, от интенсивности другого приходящего скачка

\section{Анализ области неоднозначности решения и гистерезиса}

История вопроса

Наибольший вклад в изучение феномена гистерезиса и исследование двух критериев перехода от РИ к МИ и обратно внесли сотрудники ИТПМ $[122,124]$, а также Бен-Дор [124]. Стоит отметить их совместную работу [125], в которой изучались асимметричные случаи взаимодействия встречных скачков и были изложены все основные факты, известные на тот момент. В этих работах было показано, что гистерезис существует как при изменении угла клина, так и при изменении числа Маха.

Но и в численных, и физических экспериментах моменты переключения от РИ к МИ и обратно заметно отличались от теоретических значений, предсказываемых критериями отсоединения и СМК, поэтому исследования были продолжены. В докторской диссертации Е.И. Васильева [126] гистерезис исследован численным методом с выделением разрывов. Показано, что увеличение густоты разностной 
сетки приближает численные результаты к результатам, полученным аналитически в соответствии с критериями отсоединения и СМК. Однако не было определено, сходится ли решение к предсказанному теорией или к какому-то другому. Кроме того, в ряде расчетов при помощи консервативных разностных схем получен парадоксальный результат - переход от РИ к МИ происходил ниже линии, соответствующей критерию фон Неймана. Но в этой области условия динамической совместности, полученные из законов сохранения, запрещают существование РИ, а условие консервативности разностной схемы подразумевает строгое выполнение законов сохранения. Стало очевидно, что на результаты численного решения существенно влияет схемная вязкость и порядок аппроксимации используемой в расчетах разностной схемы.

В диссертации Д.В. Хотяновского [127] явление гистерезиса было исследовано с помощью разностных схем ENO и WENO повышенного класса точности. Полученные результаты демонстрируют более узкий диапазон гистерезиса, чем предсказывает теория. И если переключение от МИ к РИ располагается близко от кривой, соответствующей критерию СМК, то отличие при переходе от РИ к МИ в соответствии с критерием отсоединения намного больше.

Исследования Д.В. Хотяновского нашли свое развитие в докторской диссертации А.Н. Кудрявцева [128], в которой численный метод, математическая модель и описание самого явления приведены наиболее полно. Исследованы случаи как симметричного, так и асимметричного взаимодействия встречных скачков. Изучено влияние скорости изменения параметров взаимодействия на ширину зоны гистерезиса. Экспериментально подтвержден факт наличия гистерезиса в области двузначности решения. Показано, что искривление скачков уплотнения при быстром изменении угла клина приводит к существенному влиянию на ширину области гистерезиса. Вопрос о влиянии скорости изменения числа Маха при фиксированном угле клина не изучался.

И наконец, в диссертации Г.В. Шоева [129] исследовано влияние размытия скачков на решение задачи об их интерференции. Для этого использован прямой метод статистического моделирования решения кинетического уравнения Больцмана. Показано, что размытие скачков в результате действия схемной или физической вязкости приводит к размыванию ударных поляр, при этом основная и вторичная поляры заменяются некоторой огибающей. В результате решение, соответствующее пересечению поляр, смещается в точку, в которой происходит перегиб огибающей.

Таким образом, проведенные ранее исследования подтвердили наличие гистерезиса и близость получаемых в численных расчетах и экспериментах моментов перехода РИ ↔ МИ к предсказанным теорией значениям. Выявлено влияние схемной и физической вязкости на ширину зоны гистерезиса.

\section{Анализ области неоднозначности}

Для области неоднозначности решения, в которой возможны нестационарные и гистерезисные явления, на рис. 18 приведены границы, образованные линиями $J_{R}(M), J_{0}(M)$. С ростом числа Маха область неоднозначности решения расширяется. Напомним, что при $M<M_{0 R}$ CMК существовать не может, и $J_{0}$ здесь не определена.

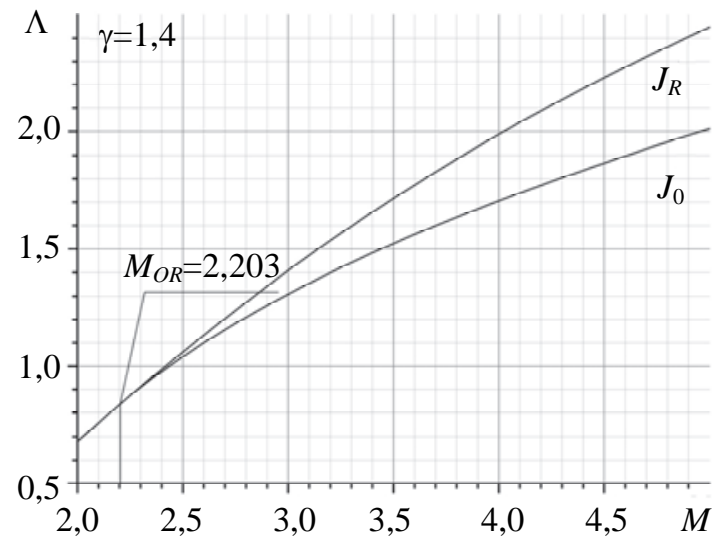

Рис. 18. Область неоднозначности, ограниченная линиями $J_{R}(M), J_{0}(M)$, в которой возможно как регулярное, так и маховское отражение скачка от стенки

Для практического использования полезнее построение области неоднозначности в координатах $M-\beta$. Действительно, в полете при разгоне летательного аппарата изменяется число Маха, а угол клина воздухозаборника остается постоянным. Наоборот, при регулировании воздухозаборника меняется угол клина, а число Маха полета, как правило, остается постоянным. Если последовательно в формулах (15)-(16) менять число Маха, то можно для каждого значения $M$ вычислить $J_{0}$ и $J_{R}$ и соответствующие им углы разворота потока $\beta$. Таким образом, можно построить на плоскости $M-\beta$ параметрические кривые: $M_{0}(\beta)$, соответствующую критерию СМК, и $M_{R}(\beta)$, соответствующую критерию отсоединения. 
Вся плоскость параметров $M-\beta$ может быть разбита на три области: выше кривой $M_{0}$, ниже кривой $M_{R}$ и между ними (рис. 19). Кривая $M_{0}$ соответствует критерию СМК. Между кривыми $M_{0}$ и $M_{R}$ вторичная поляра пересекается как с основной полярой, так и с осью ординат, т.е. условиями динамической совместности допускается как РИ, так и МИ. Это - зона неоднозначности решения. На рис. 19 видно, что существует предельный минимальный угол клина $\beta_{\min }$, при котором линии $M_{0}$ и $M_{R}$ сливаются. Этой точке соответствует особое число Маха $-M_{0 R}$. Соответственно при $\beta<\beta_{\min }$ гистерезис существовать не может, ниже линии $M_{0 R}$ лежит область МИ, выше - РИ. Переход от МИ к РИ при увеличении числа Маха происходит на линии $M_{0 R}$. На рис. 19 также хорошо видно, что у линии $M_{0}$ имеется и второй предельный угол $\beta_{\max }$. Существует также угол $\beta_{\infty}$, к которому стремится линия $M_{0}$, при $M \rightarrow \infty$. Значения особых чисел Маха $M_{0 R}$ и особых углов $\beta_{\min }, \beta_{\max }, \beta_{\infty}$ приведены в табл. 1 для $\gamma=1,1$ (смесь паров углеводородного топлива с воздухом); $\gamma=1,25$ (продукты сгорания углеводородного топлива в воздухе); $\gamma=1,4$ (двухатомный газ, например, воздух); $\gamma=1,67$ (одноатомный газ, например, водород).

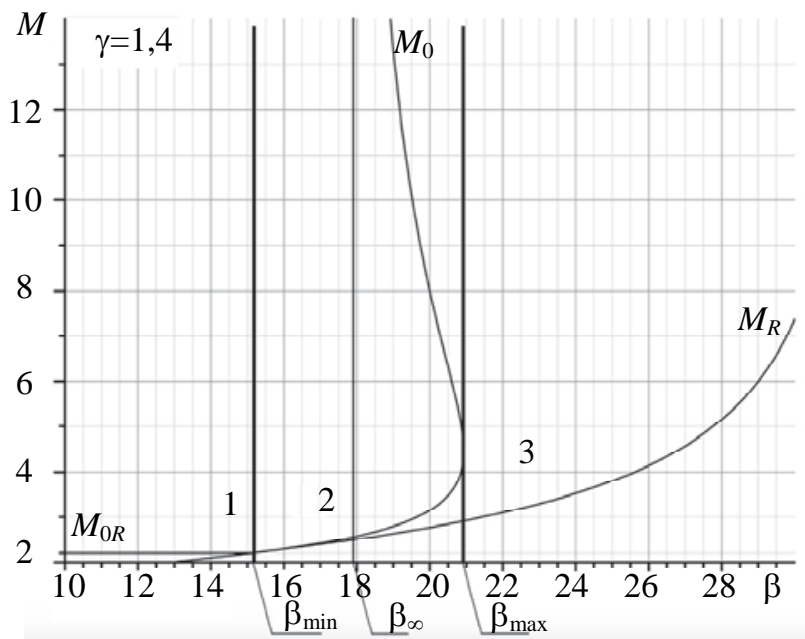

Рис. 19. Области существования РИ и МИ. 1 - область, в которой $J_{0}$ не определена, т.е. вторичная поляра не пересекает основную поляру в ее вершине ни при каких значениях интенсивности скачка; 2 - область гистерезиса; 3 - область, в которой гистерезиса нет и переход РИ↔МИ происходит при $\mathrm{J}=J_{R}$

\begin{tabular}{|c|c|c|c|c|}
\hline$\gamma$ & 1,67 & 1,4 & 1,25 & 1,1 \\
\hline$M_{0 R}$ & 2,447 & 2,203 & 2,078 & 1,952 \\
\hline$\beta_{\min }$, град & 14,5 & 15,2 & 15,6 & 15,7 \\
\hline$\beta_{\max }$ град & 19,18 & 20,921 & 22 & 23,23 \\
\hline$\beta_{\infty}$, град & 18,7 & 17,961 & 16 & 13 \\
\hline
\end{tabular}

Таблица 1. Особые значения чисел Маха и углов разворота потока

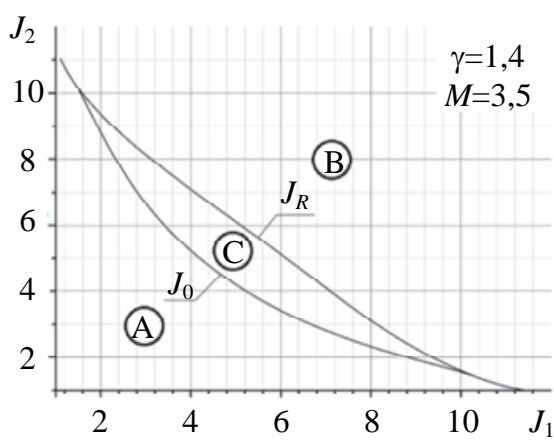

a

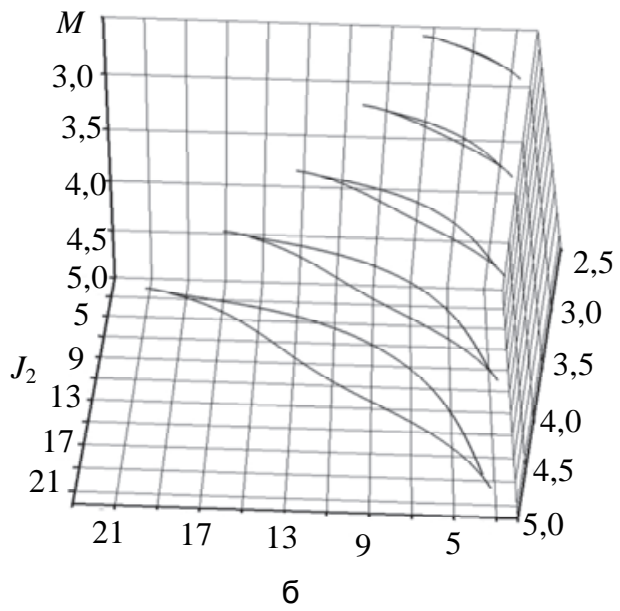

б

Рис. 20. Область неоднозначности при несимметричном взаимодействии встречных скачков: A - регулярное отражение; В - маховское отражение; C - область неоднозначности (a); области неоднозначности при различных числах Маха (б) 
Действительно, при углах клина больше $21^{\circ}$ существуют только две области: МИ ниже $M_{R}$ и область неоднозначности выше $M_{R}$. Никакого гистерезиса, вроде бы, быть не должно, и переключение от МИ к РИ должно происходить на линии $M_{R}$. Таким образом, вся плоскость разбита на три диапазона углов $\beta$ : $1-\beta<\beta_{\min }, 2-\beta_{\min }<\beta<\beta_{\max }, 3-\beta>\beta_{\max }$. В диапазоне 1 ниже линии $M_{0 R}$ реализуется МИ, выше РИ, в диапазоне 2 ниже $M_{0 R}-$ МИ, выше $M_{0}-$ РИ, между этими кривыми лежит зона гистерезиса. С увеличением показателя адиабаты области неоднозначности значительно сужаются.

Границы области неоднозначности, заключенные между линиями, соответствующими критериям фон Неймана и СМК, для случая несимметричной интерференции ВСУ приведены на рис. 20. Видно, что с увеличением числа Маха область неоднозначности расширяется (рис. 20, б).

Результаты численного анализа гистерезиса в зоне неоднозначности решения

Моделирование было выполнено в двухмерной расчетной области, расположенной между двумя клиньями [130]. Изменялось число Маха набегающего потока. Поскольку в задачах расчета струйных течений с ударными волнами выбор модели турбулентности может оказать существенное влияние на геометрию УВС [131], численное моделирование было выполнено в рамках модели идеального газа. Дополнительным доводом в пользу использования модели идеального газа служит тот факт, что переход РИ↔МИ может сопровождаться быстрой скачкообразной перестройкой УВС, т.е. существенно нестационарным процессом. В моделях же турбулентности используется осреднение турбулентного потока по времени, поэтому их применение при моделировании быстро протекающих процессов теоретически не обосновано [132].

Расчеты выполнялись на четырех различных структурированных разностных сетках. Самая грубая сетка имела 60 ячеек поперек течения, остальные - 240, 480 и 960 ячеек. Момент перехода к маховскому отражению контролировался визуально по наличию двух тангенциальных разрывов за ножкой Маха (по изолиниям полного давления $P_{0}$ и чисел Маха) и, дополнительно, по изменению кривизны отраженного скачка уплотнения. Как следует из решения задачи первого порядка об интерференции скачков, при регулярном пересечении скачков отраженный скачок имеет положительную кривизну, а при образовании тройной точки кривизна скачком изменяется на отрицательную [133]. Изменение знака кривизны происходит скачком и хорошо заметно даже тогда, когда визуально ножка Маха и тангенциальные разрывы на ней не видны.

Результаты сведены в табл. 2. Видно, что численные результаты по мере измельчения сетки в точности сходятся к теоретическим значениям.

По мере увеличения числа Маха переход к регулярному отражению происходит в соответствии с критерием СМК, при уменьшении числа Маха - в соответствии с критерием фон Неймана. Скорость изменения числа Маха не влияет на границы области гистерезиса.

\begin{tabular}{|c|c|c|c|c|}
\hline \multicolumn{3}{|c|}{} & \multicolumn{2}{c|}{ Аналитическое решение с учетом размазывания } \\
скачка \\
\hline Количество ячеек & РИ $\rightarrow$ МИ & РИ МИ & РИ $\rightarrow$ МИ & РИ МИ \\
\hline 60 & $M=2,6$ & $M=2,7$ & $M=2,612-2,975$ & $M=2,745-$ нет \\
\hline 240 & $M=2,7$ & $M=2,8$ & $M=2,715-2,835$ & $M=2,978-3,436$ \\
\hline 480 & $M=2,75$ & $M=3,0$ & $M=2,753-2,789$ & $M=3,092-3,22$ \\
\hline 960 & $M=2,8$ & $M=3,15$ & $M=2,762-2,78$ & $M=3,12-3,185$ \\
\hline $\begin{array}{c}\text { Теоретическое } \\
\text { значение }\end{array}$ & $M=2,77$ & $M=3,149$ & $M=2,77$ & $M=3,149$ \\
\hline
\end{tabular}

Таблица 2. Диапазон гистерезиса

\section{Заключение}

В статье приведен обзор работ по тройным конфигурациям ударных волн и интерференции встречных скачков уплотнения. Практика проектирования современных сверхзвуковых и гиперзвуковых воздухозаборников с внутренним и комбинированным сжатием потребовала дополнительного изучения интерференции встречных скачков, а также отражения скачков от стенки в условиях неоднозначности решения, допускающего как регулярную, так и нерегулярную интерференцию. Нерегулярная интерференция происходит с образованием тройных конфигураций ударных волн. Рассмотрены области существования тройных конфигураций ударных волн трех типов. Выполнен анализ критериев перехода от маховской интерференции к регулярной и обратно. Переход от маховской интерференции к регулярной происходит всегда в соответствии с критерием стационарной маховской конфигурации, а обратный - в соответствии с критерием отсоединения фон Немана. Физическая и схемная вязкость приводит к суже- 
нию области неоднозначности решения и уменьшению гистерезиса. Причиной является размазывание скачков. При увеличении числа ячеек разностной сетки численное решение для моментов перехода от регулярной интерференции к маховской и обратно в точности сходится к теоретическим критериям фон Неймана и стационарной маховской конфигурации.

\section{Литература}

1. Усков В.Н. Наука о стихиях. СПб.: БГТУ, 2007. 140 с.

2. Усков В.Н. Этапы становление аэрогазодинамики / В сб. Аэродинамика. Под ред. Р.Н. Митрошина. СПб.: ВВМ, 2006. С. 153-211.

3. Усков В.Н., Мостовых П.С. Исторический очерк развития исследований газодинамических разрывов / В сб. Аэродинамика. Под ред. Р.Н. Мирошина. СПб.: СПбГУ, 2013. С. 131-156.

4. Матвеев С.К., Усков В.Н. Исаак Павлович Гинзбург. К 100-летию со дня рождения // Вестник СПбГУ. Серия 1. Математика. Механика. Астрономия. 2010. № 3. С.132-137.

5. Матвеев С.К., Усков В. Н. Виктор Георгиевич Дулов. К 80-летию со дня рождения // Вестник СПбГУ. Серия 1. Математика. Механика. Астрономия. 2010. № 3. С. 138-141.

6. Handbook of Shock Waves / Eds G. Ben-Dor, O. Igra, T. Elperin. Academic Press, 2001. V. 1-3.

7. Дейч М.Е. Техническая газодинамика. Изд. 3-е. М.: Энергия, 1974. 592 с.

8. Mach E. Weitere ballistisch-photografische Versuche // Akad.Wiss.Wien. 1889. V. 98. P. 1310-1326.

9. $\quad$ Lagrange J.L. Mecanique Analytique. Paris, 1788.

10. Poisson S.D. Memoire sur la theorie du son // Journal de l'Ecole Polytechnique. 1808. V. VII. N 14. P. 319-392.

11. Stokes G.G. On a difficulty in the theory of sound / In: Classic Papers in Stock Compression Science. Eds. J.N. Johnson, R. Cheret. Springer, 1998. P. 71-81. doi: 10.1007/978-1-4612-2218-7_2

12. Арнольд В.И. Геометрические методы в теории обыкновенных дифференциальных уравнений. Ижевск: МЦНМО, 2000. 384 с.

13. Арнольд В.И. Дополнительные главы теории обыкновенных дифференциальных уравнений. М.: Наука, 1978. 304 c.

14. Zeldovich Ya.B. Gravitational instability: an approximate theory for large density perturbations // Astronomy and Astrophysics. 1970. V. 5. N 1. P. 84-89.

15. Карман Т., Бюргерс И. Теоретическая аэродинамика идеальных жидкостей. М.-Л.: Оборонгиз, 1939. 408 c.

16. Earnshaw S. On the mathematical theory of sound // Proceedings of Royal Society of London. 1858. P. 590-591.

17. Earnshaw S. On the mathematical theory of sound // Philosophical Transactions of the Royal Society of London. 1860. V. 150. N 8. P. 133-148. doi: 10.1098/rstl.1860.0009

18. Bulat P.V., Bulat M.P. Gas-dynamic variable relation on opposite sides of the gas-dynamic discontinuity // Research Journal of Applied Sciences, Engineering and Technology. 2015. V. 9. N 12. P. 1097-1104.

19. Усков В.Н. Интерференция стационарных газодинамических разрывов // Сборник статей "Сверхзвуковые газовые струи". Новосибирск: Наука, 1983. С. 22-46.

20. Усков В.Н., Тао Ган, Омельченко А.В. О поведении газодинамических переменных за косой ударной волной // Сборник статей "Современные проблемы неравновесной газо- и термодинамики". 2002. С. 179-191.

21. Усков В.Н. Ударные волны и их взаимодействие. Л.: Ленингр. мех. ин-тут, 1980. 88 с.

22. Riemann B. Uber die Fortpflanzung ebener Luftwellen von endlicher Schwingweite // Abhandlungen der koniglichen Gesellschaft der Wissenschaften zu Gottingen. 1860. V. 8. P. 43.

23. Rankine W.J.M. On the thermodynamic theory of waves of finite longitudinal disturbance // Proceedings of the Royal Society of London. 1869. V. 18. P. 80-83.

24. Rankine W.J.M. On the thermodynamic theory of waves of finite longitudinal disturbance // Philosophical Magazine. 1870. V. 39. N 4. P. 306-309.

25. Rankine W.J.M. On the thermodynamic theory of waves of finite longitudinal disturbance // Philosophical Transactions of the Royal Society of London. 1870. V. 160. P. 277-288.

26. Hugoniot H. Propagation du mouvement dans les corps. Chapitre V. Sur les discontinuités qui se manifestent dans la propagation du movement // Journal de l’École Polytechnique. 1889. V. LVIII. P. 68-125.

27. Meyer Th. Ueber zweidimensionale Bewegungsvorg ä nge in einem Gas, dasmit Ueberschallgeschwindigkeit strömt // Forschungsheft des Vereins deutcher Ingenieure. 1908. V. 62. P. 31-67.

28. У Усков В.Н. Бегущие одномерные волны. СПб.: БГТУ "ВОЕНМЕХ", 2000. 224 с.

29. Адрианов А.Л., Старых А.Л., Усков В.Н. Интерференция стационарных газодинамических разрывов. Новосибирск: Наука, 1995. 180 с.

30. Усков В.Н., Чернышев М. В. Экстремальные ударно-волновые системы в задачах внешней сверхзвуковой аэродинамики // Теплофизика и аэромеханика. 2014. Т. 21. №1. С. 15-31. 
31. Усков В.Н. Оптимальные бегущие по потоку газа одномерные волны // XV Сессия Международной школы по моделям механики сплошной среды. Санкт-Петербург, 2000. С. 63-78.

32. Усков В.Н., Мостовых П.С. Экстремальные свойства косой ударной волны, бегущей по потоку газа. Четвертые Поляховские чтения: Избранные труды Международной научной конференции по механике. Санкт-Петербург, 2006. С. 444-454.

33. Vieille P. Sur les discontinuiti és produites par la det énte brusque de gaz comprim és // Comptes Rendus. 1899. V. CXXIX. P. 1228-1230.

34. Stodola A. Beitrag zur Stromung von Gasen und D ä mpfen durch Rohre mit veranderlichem Querschnitt // Zeitschrift des Vereins deutcher Ingenieure. 1903. V. 47. P. 1787-1788.

35. Усков В.Н. Интерференция газодинамических и тангенциальных разрывов // Изв. АН СССР МЖГ. 1979. №4. C. 191-197.

36. Schardin H. Bemerkungen zum Druckausgleichsvorgang in einer Rohrleitung // Physik. Zeits. 1932. V. 33. P. 60-64.

37. Bitondo D., Glass I.I., Patterson G.N. One Dimensional Theory of Absorption and Amplification of a Plane Shock Wave by a Gaseous Layer. University of Toronto Institute of Aerophysics (UTIA), 1950. Report N5.

38. Bitondo D. Lobb R.K. Experiments on the Amplification of a Plane Shock Wave. University of Toronto Institute of Aerophysics (UTIA), 1950. Report N7.

39. Ford C.A., Glass I.I. An experimental study of one-dimensional shock wave refraction // Journal of Aerospace Sciences. 1956. V. 23. N 2. P. 189-191.

40. Gould D.G. The Head-On Collision of Two Shock Waves and a Shock and Rarefaction Wave in OneDimensional Flow. University of Toronto Institute for Aerospace Studies (UTIA), 1952. Report N17.

41. Nicholl C.I.H. The Head-On Collision of Shock and Rarefaction Waves. University of Toronto Institute of Aerophysics (UTIA), 1951. Report N10.

42. Billington I.I., Glass I.I. On the One-Dimensional Refraction of a Rarefaction Wave at a Contact Surface. University of Toronto Institute of Aerophysics (UTIA), 1955. Report N 31.

43. Billington I.I. An experimental study of one-dimensional refraction of a rarefaction wave at a contact surface // Journal of Aeronautical Sciences. 1956. V. 23. N 11. P. 997-1006.

44. Taub A.H. Refraction of plane shock waves // Physical Review. 1947. V. 72. N 1. P. 51. doi: 10.1103/PhysRev.72.51

45. Архипова Л.П., Усков В.Н. Универсальное решение задачи об отражении одномерных бегущих волн от твердой стенки и его анализ для волн уплотнения // Вестник СПбГУ. Серия 1. Математика. Механика. Астрономия. 2013. № 2. С. 77-81.

46. Архипова Л.П., Усков В.Н. Отражение центрированной волны разрежения Римана со сверхзвуковым задним фронтом от вертикальной твердой и гладкой поверхности // Вестник СПбГУ. Серия 1. Математика. Механика. Астрономия. 2012. № 4. С. 62-65.

47. Uskov V.N., Chernyshov M.V. The interaction of Prandtl-Meyer wave with the oblique shock of the same direction // Journal of Energy and Power Engineering. 2014. V. 8. P. 121-136.

48. Uskov V.N., Chernyshov M.V. Analytical solutions for overtaking Prandtl-Meyer wave-oblique shock // Proc. 19 $9^{\text {th }}$ International Shock Interaction Symposium (ISIS). Moscow, 2010.

49. Усков В.Н., Мешков В.Р., Омельченко А.В. Взаимодействие скачка уплотнения со встречной волной разрежения // Вестник СПбГУ. Серия 1. Математика. Механика. Астрономия. 2002. № 2.

50. Silnikov M.V., Chernyshov M.V., Uskov V.N. Analytical solutions for Prandtl-Meyer wave-oblique shock overtaking interaction // Acta Astronautica. 2014. V. 99. N 1. P. 175-183. doi: 10.1016/j.actaastro.2014.02.025

51. Усков В.Н., Карасев К.А. Критериальные интенсивности при интерференции прямого скачка уплотнения и встречной ударной волны / В сб. Наука и технологии. 2003. С. 4-11.

52. Омельченко А.В., Усков В.Н. Интерференция нестационарных косых ударных волн // ПЖТФ. 2002. T. 28. № 12. C. 5-12.

53. Усков В.Н., Мостовых П.С. Тройные конфигурации бегущих ударных волн в потоках невязкого газа // Прикладная механика и техническая физика. 2008. Т. 49. №3. С. 3-10.

54. Осватич К., Шварценбергер Р. Сборник задач и упражнений по газовой динамике. М.: Мир, 1967.

55. Усков В.Н., Омельченко А. В. Оптимальные ударно-волновые системы // Изв. РАН. МЖГ. 1995. №6. С. 126-134.

56. Усков В.Н., Омельченко А.В. Управление сверхзвуковым потоком. Доклад к 90-летию со дня рождения профессора Н.Н. Поляхова, 1997.

57. Омельченко А.В., Усков В.Н. Геометрия оптимальных ударно-волновых систем // Прикладная механика и техническая физика. 1997. Т. 38. №5. С. 29-35.

58. Малоземов В.Н., Омельченко А.В., Усков В. Н. О минимизации потерь полного давления при торможении сверхзвукового потока // Прикладная математика и механика. 1998. Т. 62. №6. С. 1014-1021. 
59. Омельченко А.В., Усков В.Н. Оптимальные ударно-волновые системы при ограничениях на суммарный угол поворота потока // Изв. РАН. МЖГ. 1996. №4. С. 142-150.

60. Омельченко А.В., Усков В.Н. Максимальные углы поворота сверхзвукового потока в ударноволновых системах // Изв. РАН. МЖГ. 1998. №3. С. 148-156.

61. Омельченко А.В., Усков В.Н. Оптимальные догоняющие скачки уплотнения с ограничениями на суммарный угол поворота потока // Прикладная механика и техническая физика. 1999. Т. 40. № 4. C. 99-108.

62. Омельченко А.В., Усков В.Н. Экстремальная система волна разрежения - скачок уплотнения в стационарном потоке газа // Прикладная механика и техническая физика. 1997. Т. 38. №2. С. 40-47.

63. Uskov V.N., Chernyshov M.V., Erofeev V.K., Genkin P. Optimal shock-wave structures and new ideas about supersonic gas jet noise generation // Proc. $13^{\text {th }}$ Int. Congress on Sound and Vibration. Vienna, Austria, 2006. P. 1439-1446.

64. Усков В.Н., Чернышов М.В. Теоретический анализ аэродинамических коэффициентов многоугольных профилей в сверхзвуковом потоке, механика и процессы управления // Труды ХХХІ Уральского семинара. Екатеринбург, 2001. С. 187-191.

65. Усков В.Н., Чернышев М.В. Анализ аэродинамических коэффициентов многоугольных профилей в сверхзвуковом потоке // Сб. трудов X Всероссийского семинара по управлению движением и навигации летательных аппаратов. Самара, 2002. С. 322-326.

66. Усков В.Н., Чернышев М.В. Экстремальные нагрузки на элементы конструкций, доставляемые ударно-волновыми системами // Труды 8-й Всероссийской конференции "Проблемы обеспечения взрывобезопасности и противодействия терроризму". Санкт-Петербург, 2013. С. 203-226.

67. Усков В.Н., Чернышев М.В. Тройные конфигурации стационарных ударных волн // XII Всероссийская научно-практическая конференция "Актуальные проблемы защиты и безопасности". СанктПетербург, 2009. С. 420-435.

68. Усков В.Н., Шахова О.А. К расчету тройной конфигурации ударных волн // Гидроаэромеханика и теория упругости. 1976. №21. С. 13-18.

69. Мельников Д.А. Отражение скачков уплотнения от оси симметрии // Изв. АН СССР Механика и машиностроение. 1962. №3. С. 24-30.

70. Рылов А.И. К вопросу о невозможности регулярного отражения стационарной ударной волны от оси симметрии // Прикладная математика и механика. 1990. Т. 25. № 2. С. 245-249.

71. Исакова Н.П., Крайко А.Н., Пьянков К.С., Тилляева Н. И. Об усилении слабых ударных волн в осесимметричном сверхзвуковом потоке и их отражений от оси симметрии // Прикладная математика и механика. 2012. Т. 76. № 4. С. 623-645.

72. von Neumann J. Oblique reflection of shocks / In: Collected Works, Pergamon. 1943. V. 6. P. 239-299.

73. Courant R., Friedrichs K.O. Supersonic Flow and Shock Waves. NY: Interscience, 1948.

74. Bleakney W., Fletcher C.H., Weimer D.K. The density field in mach reflection of shock waves // Physical Review. 1949. V. 76. N 2. P. 323-324. doi: 10.1103/PhysRev.76.323.2

75. Breed B.R. Impossibility of three confluent shocks in two-dimensional irrotational flow // Physics of Fluids. 1967. V. 10. N 1. P. 21-23. doi: 10.1063/1.1761977

76. Sternberg J. Triple-shock-wave intersections // Physics of Fluids. 1959. V. 2. N 2. P. 179-206. doi: 10.1063/1.1705909

77. Sakurai A. On the problem of weak Mach reflection // Journal of the Physical Society of Japan. 1964. V. 19. N 8. P. 1440-1450. doi: 10.1143/JPSJ.19.1440

78. Dulov V.G. Motion of triple configuration of shock waves with formation of wake behind branching point // Journal of Applied Mechanics and Technical Physics. 1973. V. 14. N 6. P. 791-797. doi: 10.1007/BF00853193

79. Дулов В.Г., Лукьянов Г.А. Газодинамика процессов истечения. Новосибирск: Наука, 1984. 236 с.

80. Усков В.Н., Чернышов М. В. Теоретический анализ особенностей тройных конфигураций скачков уплотнения // В сб. Современные проблемы неравновесной газо- и термодинамики. СанктПетербург: БГТУ, 2002. С. 75-99.

81. Росляков Г.С., Старых А.Л., Усков В.Н. Интерференция стационарных скачков уплотнения одного направления. // Изв. АН СССР. Механика жидкости и газа. 1987. № 4. С. 143-152.

82. Усков В.Н., Старых А.Л. Анализ областей существования решений уравнений интерференции стационарных газодинамических разрывов / В сб. Нестационарные течения газов с ударными волнами. Ленинград, 1990. С. 359-372.

83. Усков В.Н., Адрианов А.Л. Комплекс подпрограмм для моделирования двумерных стационарных сверхзвуковых течений с выделением разрывов на несогласованной сетке / В сб. Комплексы программ математической физики и архитектура ЭВМ. 1988. С. 3-7.

84. Усков В.Н., Чернышев М.В. Особые и экстремальные тройные конфигурации скачков уплотнения // Прикладная механика и техническая физика. 2006. Т. 47. №4. С. 39-53. 
85. Усков В.Н., Чернышев М.В. Анализ и оптимизация ударно-волновой структуры сверхзвуковых газовых струй // IX Всероссийский съезд по теоретической и прикладной механике. Нижний Новгород, 2006.

86. Усков В.Н., Чернышов М.В. Особые и оптимальные свойства стационарных Маховских конфигураций // Известия ТулГУ. Проблемы специального машиностроения. 2001. Вып. 4, Ч.1. С. 216-220.

87. Uskov V.N., Mostovykh P.S., Chernyshov M.V. Special and extreme structures of stationary and nonstationary shocks // Proc. $18^{\text {th }}$ Int. Shock Interaction Symposium. Rouen, 2008. P. 71-74.

88. Uskov V.N., Mostovykh P.S. Triple configurations of traveling shock waves in inviscid gas flows // Journal of Applied Mechanics and Technical Physics. 2008. V. 49. N 3. P. 347-353. doi: 10.1007/s10808-0080048-9

89. Law C.K., Glass I.I. Diffraction of strong shock waves by a sharp compressive corner // CASI Trans. 1971. V. 4. N 1. P. 2-12.

90. Ben-Dor G. Regions and transitions of nonstationary oblique shock-waves diffractions in perfect and imperfect gases // Toronto University Institute for Aerospace Studies Report. 1978. N 232.

91. Lee J.-H., Glass I.I. Pseudo-stationary oblique-shock-wave reflections in frozen and equilibrium air // Progress in Aerospace Sciences. 1984. V. 21. P. 33-80. doi: 10.1016/0376-0421(84)90003-4

92. Mostovykh P.S., Uskov V.N. Triple-shock-wave configurations: comparison of different thermodynamic models for diatomic gases // Proc. $28^{\text {th }}$ International Symposium on Shock Waves. 2012. V. 2. P. 945-951. doi: 10.1007/978-3-642-25685-1_144

93. Mach E. Uber den verlauf von funkenwellen in der ebene und im raume // Sitzungsber. Akad. Wiss. Wien. 1878. V. 78. P. 819-838.

94. Neumann J. Collected Works. Pergamon Press, 1963. 795 p.

95. Smith L.G. Photographic investigations of the reflection of plane shocks in air // Office of Scientific Research and Development, 6271. NDRC Rep. A-350. 1945.

96. White D.R. An Experimental Survey of the Mach Reflection of Shock Waves. Princeton University, Department of Physics, 1951.

97. Ben-Dor G. Shock Wave Reflection Phenomena. $2^{\text {nd }}$ ed. Springer, 2007. 342 p. doi: 10.1007/978-3-54071382-1

98. Bleakney W., Taub A.H. Interaction of shock waves // Reviews of Modern Physics. 1949. V. 21. N 4. P. 584-605. doi: 10.1103/RevModPhys.21.584

99. Bargmann V. On nearly glancing reflection of shocks // Office Sci. Res. Develop. Rep. No. 5117, 1945.

100. Lighthill M.J. The diffraction of blast // Proceedings of the Royal Society of London, Series A. 1949. V. 198. P. 454-470.

101. Ting L., Ludloff H.F. Aerodynamics of blasts // Journal of Aeronautical Sciences. 1951. V. 18. P. 143.

102. Fletcher C.H., Bleakney W. The Mach reflection of shock waves at nearly glancing incidence // Reviews of Modern Physics. 1951. V. 23. N 3. P. 271-286. doi: 10.1103/RevModPhys.23.271

103. Henderson L.F., Siegenthaler A. Experiments on the diffraction of weak blast waves: the von Neumann paradox // Proceedings of the Royal Society of London, Series A: Mathematical and Physical Sciences. 1980. V. 369. N 1739. P. 537-555. doi: 10.1098/rspa.1980.0015

104. Colella P., Henderson L.F. The von Neumann paradox for the diffraction of weak shock waves // Journal of Fluid Mechanics. 1990. V. 213. P. 71-94. doi: 10.1017/S0022112090002221

105. Adachi T., Suzuki T., Kobayashi S. Mach reflection of a weak shock waves // Transactions of the Japan Society of Mechanical Engineers, Part B. 1994. V. 60. N 575. P. 2281-2286.

106. Olim M., Dewey J.M. A revised three-shock solution for the Mach reflection of weak shocks // Shock Waves. 1992. V. 2. N 3. P. 167-176. doi: 10.1007/BF01414639

107. Guderley K.G. Considerations on the structure of mixed subsonic supersonic flow patterns, air materiel command // Technical Report F-TR-2168-ND. Wright Field, Dayton, 1947.

108. Guderley K.G. The Theory of Transonic Flow. Oxford, Pergamon Press, 1962. 344 p.

109. Васильев И.Е., Крайко А.Н. Численное моделирование дифракции слабых скачков на клине в условиях парадокса Неймана // Журнал вычислительной математики и математической физики. 1999. Т. 39. № 8. C. 1393-1404.

110. Vasil'ev E.I. Four-wave scheme of weak Mach shock waves interaction under the von Neumann paradox conditions // Fluid Dynamics. 1999. V. 34. N 3. P. 421-427.

111. Vasilev E., Olkhovsky M. // Proc. $27^{\text {th }}$ Int. Symposium on Shock Waves. St. Petersburg, 2009.

112. Булат П.В., Денисенко П.В. Интерференция скачков уплотнения одного направления // Научнотехнический вестник информационных технологий, механики и оптики. 2015. Т.15. №3. С. 500-508. doi: 10.17586/2226-1494-2015-15-3-500-508

113. Busemann A. Verdichtungsstoße in ebenen Gasstromungen. Berlin: Julius Springer, 1930. P. $162-169$.

114. Busemann A. Gasdynamik, Handbuch der experimentellen Physik. Leipzig: Akademischer Verlag. 1931. V. 4(1). P. 394. 
115. Busemann A. Hodographenmethode der Gasdynamik // ZAMM. 1937. V. 17. N 2. P. 73-79.

116. Kawamura R., Saito H. Reflection of shock waves-1 pseudo-stationary case // Journal of the Physical Society of Japan. 1956. V. 11. N 5. P. 584-592.

117. Bulat P.V., Uskov V.N. Mach reflection of a shock wave from the symmetry axis of the supersonic nonisobaric jet // Research Journal of Applied Sciences, Engineering and Technology. 2014. V. 8. N 1. P. 135-142.

118. Molder S. Head-on interaction of oblique shock waves. University of Toronto Institute for Aerospace Studies Technical Note. 1960. N 38.

119. Булат П.В., Денисенко П.В., Упырев В.В. Несимметричное взаимодействие встречных косых ударных волн // Научно-технический вестник информационных технологий, механики и оптики. 2015. T.15. №5. C. 942-949. doi: 10.17586/2226-1494-2015-15-5-942-949

120. Булат П.В., Упырев В.В., Денисенко П.В. Отражение косого скачка уплотнения от стенки. // Научно-технический вестник информационных технологий, механики и оптики. 2015. Т.15. №2. С. 338345. doi: 10.17586/2226-1494-2015-15-2-338-345

121. Старых А.Л. Нерегулярное взаимодействие скачков уплотнения между собой и с тангенциальными разрывами // Численные методы механики сплошной среды. 1986. Т. 17. № 6. С. 119-124.

122. Fomin V.M., Hornung H.G., Ivanov M.S., Kharitonov A.M., Klemenkov G.P., Kudryavtsev A.N., Pavlov A.A. The study of transition between regular and Mach reflection of shock waves in different wind tunnels // Proc. $12^{\text {th }}$ Int. Mach Reflection Symposium. Pilanesberg, South Africa, 1996. P. 137-151.

123. Ivanov M.S., Ben-Dor G., Elperin T., Kudryavtsev A., Khotyanovsky D. Flow-Mach-number-variationinduced hysteresis in steady flow shock wave reflections // AIAA Journal. 2001. V. 39. N 5. P. 972-974. doi: $10.2514 / 2.1406$

124. Ben-Dor G. Two-dimensional interactions / In: Handbook of Shock Waves / Eds G. Ben-Dor, O. Igra, T. Elperin. Boston: Academic Press, 2001. 824 p.

125. Ivanov M.S., Ben-Dor G., Elperin T., Kudryavtsev A.N., Khotyanovsky D.V. The reflection of asymmetric shock waves in steady flows: a numerical investigation // Journal of Fluid Mechanics. 2002. V. 469. P. $71-$ 87. doi: $10.1017 / S 0022112002001799$

126. Васильев Е.И. W-модификация метода Годунова и ее приложения в моделировании газодинамических течений с ударными волнами: дис. ... д-ра физ.-мат. наук. Волгоград, 1999. 213 с.

127. Хотяновский Д.В. Численный анализ сверхзвуковых течений со сложными ударно-волновыми структурами: дис. ... канд. физ.-мат. наук. Новосибирск, 2007. 148 с.

128. Кудрявцев А.Н. Вычислительная аэродинамика сверхзвуковых течений с ударными волнами: дис. ... д-ра физ.-мат. наук. Новосибирск, 2014. 337 с.

129. Шоев Г.В. Численное исследование влияния вязкости на процессы взаимодействия и распространения ударных волн: дис. ... канд. физ.-мат. наук. Новосибирск, 2013. 134 с.

130. Булат П.В., Упырев В.В. Границы области неоднозначности при отражении скачков уплотнения // ПЖТФ. 2016. Т. 42. № 1. С. 33-41.

131. Ilina E.E., Ilina T.E., Bulat P.V. Analysis of the application of turbulence models in the calculation of supersonic gas jet // American Journal of Applied Sciences. 2014. V. 11. N 11. P. 1914-1920. doi: 10.3844/ajassp.2014.1914.1920

132. Bulat M.P., Bulat P.V. Comparison of turbulence models in the calculation of supersonic separated flows // World Applied Sciences Journal. 2013. V. 27. N 10. P. 1263-1266. doi: 10.5829/idosi.wasj.2013.27.10.13715

133. Uskov V.N., Mostovykh P.S. Differential characteristics of shock waves and triple shock wave configurations // Proc. $20^{\text {th }}$ Int. Shock Interaction Symposium (ISIS 2012). Stockholm, 2012. P. 211-214.

Булат Павел Викторович

Денисенко Петр Валерьевич

Pavel V. Bulat

Petr V. Denissenko
- кандидат физико-математических наук, кандидат экономических наук, заведующий международным научным подразделением, Университет ИТМО, Санкт-Петербург, 197101, Российская Федерация, pavelbulat@mail.ru

- $\quad \mathrm{PhD}$, доцент, Университет Уорвика, Ковентри, CV4 7AL, Великобритания, p.denissenko@gmail.com

- $\quad$ PhD, Scientific supervisor of International laboratory, ITMO University, Saint Petersburg, 197101, Russian Federation, pavelbulat@mail.ru

- $\quad$ PhD, Associate Professor, Warwick University, Coventry, CV4 7AL, United Kingdom, p.denissenko@gmail.com 